\title{
基于亚胺瞬态导向策略的过渡金属催化的碳氢键活化
}

\author{
吴勇杰史炳锋* \\ (浙江大学化学系 杭州 310027)
}

\begin{abstract}
摘要 在过去几十年, 过渡金属催化的碳氢键活化取得了巨大的发展, 并给有机合成领域带来了革命性的改变. 与传 统的通过共价连接的导向基相比, 基于瞬态导向策略的碳氢键活化, 因为规避了传统导向基需要额外的步骤进行安装 和脱除的不足，极大地提高了合成效率，拓宽了合成应用范围. 综述了近年来基于亚胺瞬态导向策略的过渡金属催化 的碳氢键活化的研究进展, 着重探讨了各种瞬态导向基及其应用. 按照底物类型和所活化碳氢键的类型进行分类, 并 对该领域目前存在的局限性和未来发展进行了总结和展望.
\end{abstract}

关键词＼cjkstart瞬态导向策略; 过渡金属; 碳氢键活化; 亚胺; 导向基

\section{Transition Metal-Catalyzed C-H Activation via Imine-Based Transient Directing Group Strategy}

\author{
Wu, Yongjie Shi, Bingfeng* \\ (Department of Chemistry, Zhejiang University, Hangzhou 310027)
}

\begin{abstract}
In the past decades, transition metal-catalyzed $\mathrm{C}-\mathrm{H}$ activation has experienced tremendous growth and revolutionized the field of organic synthesis. Several elegant strategies have been developed to promote reactivity and control precise site-selectivity. Among which, transient directing group strategy has been recognized to be an efficient and powerful approach for selective $\mathrm{C}-\mathrm{H}$ functionalization. In contrast to traditional directing groups with covalent linkage, transient directing group strategy circumvents the covalent installation and removal of directing groups, which significantly improve the synthetic efficiency and broaden the range of synthetic applications. The recent advances in imine-based transition directing groups are summarized, providing an overview of recent achievements in this cutting-edge research field over the past few years. For clarity, it is classified into two sections according to the type of substrate and the type of activated hydrocarbon bond. Emphasis is placed on the fully discussion of various transient directing groups and their applications. Finally, the limitations of previous works and perspectives on this cutting-edge area are also described.
\end{abstract}

Keywords transient directing group strategy; transition metal; $\mathrm{C}-\mathrm{H}$ activation; imine; directing groups

碳氢键是有机分子中最为普遍存在的单元, 若能实 现碳氢键的直接官能团化, 势必将极大地提高化学合成 的效率, 也为逆合成分析提供全新的思路 ${ }^{[1]}$. 但由于碳 氢键具有较高的稳定性, 且同一底物中通常存在多个化 学环境相似的碳氢键, 实现特定碳氢键的高效高选择性 活化极具挑战 ${ }^{[2]}$. 利用底物本身存在或者额外引入的配 位官能团作为导向基，与过渡金属配位，使金属催化剂 靠近并切断邻近位置的碳氢键, 通常形成较为稳定的五
元或者六元环金属中间体，最终实现特定碳氢键的选择 性活化. 因此，导向基团的存在，既能很好地实现位点 选择性，又作为配体，对催化中心金属的电子和位阻等 进行有效调控，提高反应活性. 目前，最常用的导向基 仍然是以通过共价键连接的含有氮、氧、磷、硫等强配 位能力杂原子的官能团 ${ }^{[3]}$. 通过共价键连接引入合适的 导向基虽然已经被广泛应用于选择性碳氢键官能团化 反应, 但需要额外的步骤进行引入、转化或者脱除, 降

\footnotetext{
* Corresponding author. E-mail: bfshi@zju.edu.cn

Received March 25, 2020; revised April 30, 2020; published online May 11, 2020.

Dedicated to the 40th anniversary of Chinese Journal of Organic Chemistry.

Project supported by the National Natural Science Foundation of China (Nos. 21925109, 21772170), the Outstanding Young Talents of Zhejiang Province (No. ZJWR0108), the Fundamental Research Funds for the Central Universities (No. 2018XZZX001-02) and the Natural Science Foundation of Zhejiang Province (No. LR17B020001).

国家自然科学基金(Nos. 21925109, 21772170)、浙江省万人计划青年拔尖(No. ZJWR0108)、中央高校基本科研业务费(No. 2018XZZX001-02)和浙江省 自然科学基金(No. LR17B020001)资助项目.
} 
低了合成效率，同时也限制了其在复杂分子合成中的应 用.

为了解决这一问题, 瞬态导向策略越来越多地被应 用于选择性碳氢键活化 (Scheme 1, A $)^{[4]}$. 这种瞬态导向 策略依赖于可逆共价键, 通过有机小分子催化剂与底物 原位形成新的导向基, 将原本的弱配位基团转化成强的 配位基团或把底物中不能导向的官能团转化为导向基 团(Int 1), 如将酮转化成亚胺、肜或腙. 原位生成的强配 位基团与金属配位并活化邻位碳氢键, 形成环金属中间 体 Int 2, 一般是形成热力学和动力学上更有利的五元 或六元环金属中间体. 接着发生官能团化, 生成碳氢键 官能团化的产物 Int 4. 然后原位脱除瞬态导向基团, 解 离催化金属, 得到目标产物, 同时也实现了有机小分子 和金属催化剂的催化循环. 到目前为止, 化学家们已经 成功发展了一系列基于瞬态导向策略的过渡金属催化 的碳氢键官能团化反应, 包括亚胺类、亚磷酸酯类和烯 胺类(Scheme 1, B $)^{[4-7]} .1997$ 年, Jun 课题组 ${ }^{[8]}$ 开创性地报 道了首例基于亚胺瞬态导向的铑催化醛基碳氢键烷基 化反应. Bedford 课题组 ${ }^{[5]}$ 则在亚磷酸酯类瞬态导向领域 做出贡献. 2003 年, 他们 ${ }^{[5 a]}$ 成功地实现了铑催化亚磷酸 酯瞬态导向的苯酚类化合物的邻位碳氢键芳基化. 董广 涁课题组 ${ }^{[6-7]}$ 则在烯胺瞬态导向的碳氢键活化领域做出 一系列开创性的工作. 2014 年, 他们 ${ }^{[6]}$ 报道了以催化量 胺为有机小分子催化剂的铑催化烯胺瞬态导向的酮类 衍生物 $\alpha$ 位碳氢键烷基化反应. 基于亚磷酸酯和烯胺的 瞬态导向策略已经被系统地论述过，而基于亚胺的瞬态 导向策略近年来发展迅速, 并在构建碳碳键、各类碳杂 键以及不对称碳氢键活化方面都取得了极大进展. 因 此, 撰写一篇系统而全面的综述将该领域予以概括和展 望是极有必要的. 鉴于此, 本文将从能形成亚胺导向基 的官能团入手, 分类介绍醛/酮类化合物和胺类化合物 的碳氢键官能团化反应, 详细概括截至目前为止所发展 的瞬态导向基团、所适用的反应类型以及不对称碳氢键 活化进展(图 1) ${ }^{[8-25,27,29-32,34-55,57-60,63]}$, 并对该领域的局限 性和未来的发展进行分析和展望.

\section{1 醛酮类化合物的碳氢键官能团化}

\section{1 碳碳键的构筑}

\subsection{1 醛基碳氢键的活化}

1997 年, Jun 课题组 ${ }^{[8]}$ 报道了首例铑催化烯烃与醛 的分子间氢酰化合成酮的反应(Scheme 2). 该反应的关 键在于使用催化量的 2-氨基-3-甲基吡啶(TDG1)作为金 属铑的协同催化剂——瞬态导向基. 值得一提的是, 在 这一反应体系中, 只得到反马尔科夫尼科夫规则的直链 烷基酮产物. 这可能是受金属铑、2-氨基-3-甲基吡啶

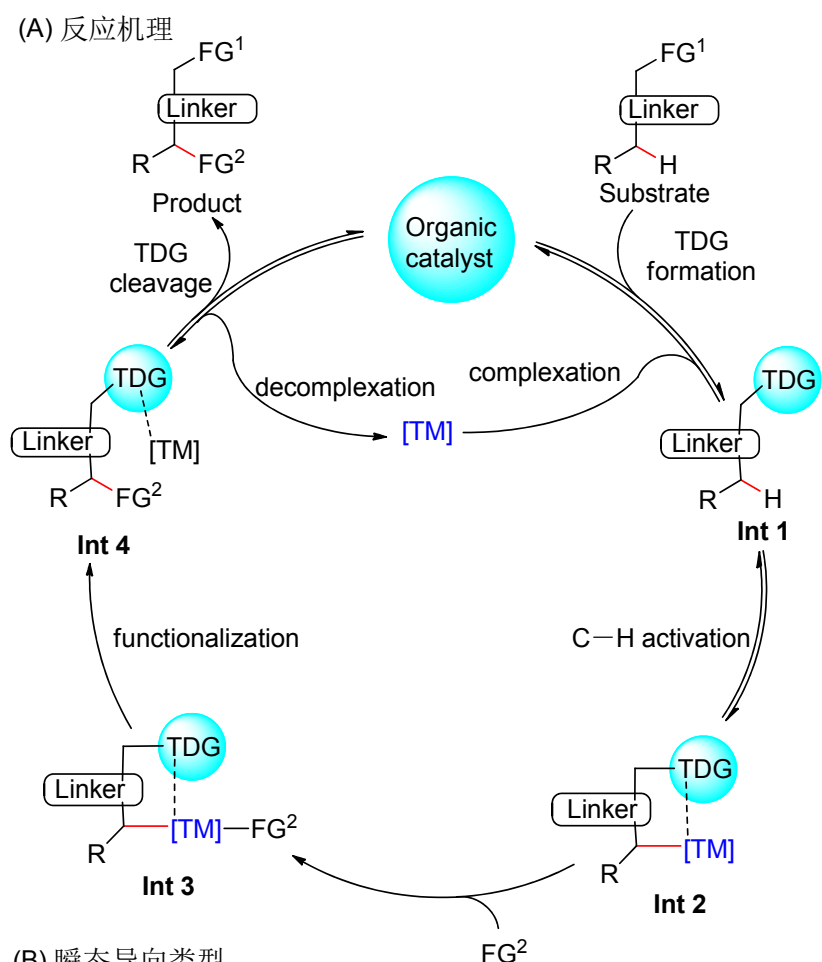

(B) 瞬态导向类型

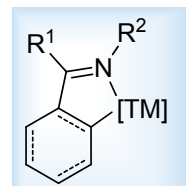

(a) 亚胺类

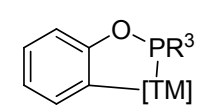

(b) 亚磷酸酯类

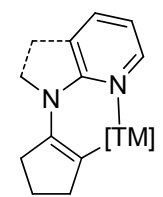

(c) 烯胺类
图式 1 基于瞬态导向策略的过渡金属催化惰性碳氢键活化 Scheme 1 Transition metal-catalyzed $\mathrm{C}-\mathrm{H}$ activation via transient directing group strategy

$\mathrm{TDG}=$ transient directing group; $\mathrm{TM}=$ transition metal

和底物醛所形成的金属中间体上的空间位阻的影响. 而 后作者还成功地将反应体系拓展到了杂环芳香醛上 ${ }^{[9]}$.

Jun et al., 1997

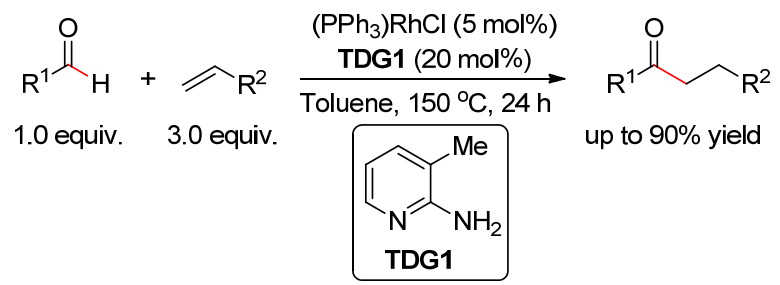

图式 2 铑催化的烯烃与醛的分子间氢酰化反应

Scheme 2 Rhodium-catalyzed intermolecular hydroacylation of 1-alkene with aldehyde

2011 年, Breit 课题组 ${ }^{[10]}$ 受 P,N 配体的启发, 在 2-氨 基-3-甲基吡啶的 6 位引入含膦基团, 发展了一种新的瞬 态导向基 TDG2, 并高效地实现了铑催化烯烃的分子间 和分子内的氢酰化反应(Scheme 3). 其中，作者认为含 膦基团的引入不但能促进形成一个高活性的氢酰化催 
(A) $\mathrm{C}-\mathrm{H}$ activation of aldehydes/ketones (Section 1)

(a) Aldehydic $\mathrm{C}-\mathrm{H}$ activation (Section 1.1.1)<smiles>Cc1cccnc1N</smiles>

TDG1

Jun, 1997, $\mathrm{Rh}(\mathrm{I})$ Hydroacylation [ref. 8-9]<smiles>Cc1ccc(Cc2ccccc2)nc1N</smiles>

Breit, 2011, $\mathrm{Rh}(\mathrm{I})$ Hydroacylation [ref. 10]<smiles>Cc1cc(N2CCCC2)cnc1N</smiles>

TDG3

Douglas, 2012, Rh(I) Hydroacylation [ref. 11]<smiles>Cc1cc(N2C(=O)OC(c3ccccc3)(c3ccccc3)C2Cc2ccccc2)cnc1N</smiles>

Douglas, 2012, $\mathrm{Rh}(\mathrm{I})$ Hydroacylation [ref. 11]*

(b) (Hetero)aryl $\mathrm{C}\left(\mathrm{sp}^{2}\right)-\mathrm{H}$ activation (Section 1.1.2)<smiles>NCc1ccccc1</smiles>

(1) Jun, 2002

$\mathrm{Rh}(\mathrm{I})$, Alkylation [ref. 12];

(2) Cheng, 2017

$\mathrm{Rh}(\mathrm{III})$, Annulation [ref. 20]<smiles>CCC(N)c1c(F)cccc1C(F)(F)F</smiles>

Wang, 2019

Rh(III), Annulation [ref. 16] ${ }^{*}$

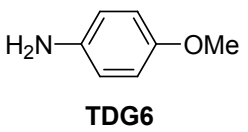

Takai, 2006

$\operatorname{Re}(\mathrm{I})$, Annulation [ref. 13]<smiles></smiles>

(1) Cheng, 2013

$\mathrm{Rh}(\mathrm{III})$, Annulation [ref. 14]; (2) Hu, 2016

$\mathrm{Pd}(\mathrm{II})$, Arylation [ref. 37a];

(3) Bao, 2020

Pd(II), Arylation [ref. 37b];<smiles>Nc1ccccc1C(=O)O</smiles>

(1) Sorensen, 2017

Pd(II), Arylation [ref. 18]

(2) Yu, 2017

$\mathrm{Pd}(\mathrm{II})$, Chlorination [ref. 17]

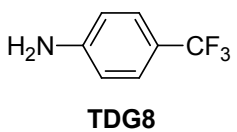

(1) Seayad, 2013

Rh(III), Annulation [ref. 15]; (2) Jiao, 2018

Rh(III), Amidation [ref. 46];

(3) Zhang, 2019

Pd(II), Chlorination [ref. 51]

Pd(II), Arylation [ref. 17]; (2) Wang, 2018

Pd(II), Arylation [ref. 22]

(3) Sorensen, 2017

Pd(II), Polyfluoroalkoxylation [ref. 50]<smiles>CC(C)(N)CC(=O)O</smiles>

Ge, 2018 Pd(II), Arylation [ref. 21]<smiles>Nc1cc(C(F)(F)F)cc(C(F)(F)F)c1</smiles>

TDG14

(1) Zhang, 2019

Pd(II), Arylation [ref. 23];

(2) $\mathrm{He}, 2017$

Ir(III), Amidation [ref. 45]<smiles>Nc1ccccc1S(=O)(=O)O</smiles>

Sorensen, 2018

$\mathrm{Pd}(\mathrm{II})$, Methylation [ref. 24]

$$
\mathrm{H}_{2} \widehat{\mathrm{NDG}}_{\mathrm{COOH}}
$$$$
\text { Jin, } 2017
$$

Pd(II), Arylation [ref. 19]

1) Shi 2017

Pd(II), Olefination [ref. 27a] ${ }^{*}$;

${ }_{\text {TDG17 }}^{t-\mathrm{Bu}}$

Pd(II), Alkynylation [ref. 29a] ; Allylation [ref. 29b]*;

(3) Shi 2019

Pd(II), Naphthylation [ref. 30]*; Alkynylation [ref. 32a]*; Allylation, Alkenylation [ref. 32b]*;

(4) Xie 2019

$\mathrm{Pd}(\mathrm{II})$, Olefination [ref. 32c]*;

(5) Jin 2018

$\mathrm{Pd}(\mathrm{II})$, Arylation [ref. 35]*<smiles>NC(C(=O)O)C(=O)O</smiles>

Xie, 2019

$\mathrm{Pd}(\mathrm{II})$, Olefination [ref. 32c]

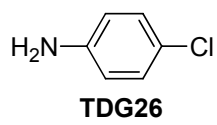

Li, 2019

Co(III), Amidation [ref. 48]<smiles>CCCCCCCCCC(C)(C)C</smiles>

Shi, 2020

$\mathrm{Pd}(\mathrm{II})$, Olefination [ref. 34]<smiles>Nc1cc(C(F)(F)F)ccc1F</smiles>

Yu, 2017

$\operatorname{Ir}($ III), Amidation [ref. 17]

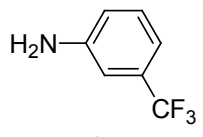

TDG24

(1) Shi, 2016

Ir(III), Amidation [ref. 44];

(2) Zhang, 2019

$\mathrm{Pd}(\mathrm{II})$, Methoxylation [ref. 51]

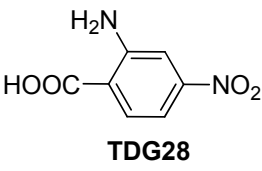

Yu, 2017

Pd(II), Bromination [ref. 17]<smiles>Cc1c(N)cccc1C(F)(F)F</smiles>

(1) Zhang, 2018

Ru(II), Alkylation [ref. 25a];

(2) Rasheed, 2018

$\mathrm{Ru}(\mathrm{II})$, Amidation [ref. 47] 
<smiles>Nc1cc(Cl)ccc1C(=O)O</smiles>

Sorensen, 2017 Pd(II), Hydroxylation Chlorination [ref. 50]

(c) $\mathrm{C}\left(\mathrm{sp}^{3}\right)$-H Activation (Section 1.1.3)

$$
\mathrm{H}_{2} \mathrm{~N} \widehat{\mathrm{COOH}}
$$$$
\text { TDG12 }
$$

(1) Yu, 2016

Pd(II), Arylation [ref. 36];

(2) Zhang, 2018

$\mathrm{Pd}(\mathrm{II})$, Arylation [ref. 39]<smiles></smiles>

Bull, 2017

$\operatorname{Pd}(\mathrm{II})$, Arylation [ref. 41]

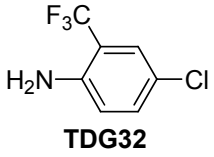

Zhang, 2019

$\mathrm{Pd}(\mathrm{II})$, Bromination [ref. 52]<smiles>CC(CO)(CO)NC(=O)C(N)Cc1ccccc1</smiles>

Kim, 2018

Pd(II), Arylation [ref. 38]<smiles>CCNC(=O)C(N)Br</smiles>

Yu, 2018

$\mathrm{Pd}(\mathrm{II})$, Fluorination [ref. 49]

.... continued $\cdot \ldots \cdot$.

(B) $\mathrm{C}-\mathrm{H}$ activation of amines (Section 2)<smiles>COC(=O)COC(=O)C=O</smiles>

Ge, 2016 Pd(II), Arylation [ref. 55]<smiles>O=Cc1c(O)cccc1Cl</smiles>

Yu,2018

$\mathrm{Pd}(\mathrm{II})$, Arylation [ref. 60]<smiles>CC(C)(C)O[Na]</smiles>

Dong, 2016

Pd(II), Arylation [ref. 57]<smiles>COc1ccccc1C(=O)C(=O)O</smiles>

Yu,2018

$\mathrm{Pd}(\mathrm{II})$, Arylation [ref. 60]<smiles>O=Cc1cccnc1O</smiles>

TDG37

(1) Yu, 2016

Pd(II), Arylation [ref. 58]

(2) Yu, 2018

$\mathrm{Pd}(\mathrm{II})$, Arylation [ref. 60]<smiles>NC(CC(=O)O)c1ccccc1</smiles>

Shi, 2020

$\mathrm{Pd}(\mathrm{II})$, Silylation [ref. 54]

\section{$\mathrm{H}_{2} \mathrm{~N} \frown \mathrm{COOH}$ \\ TDG21}

(1) Ge, 2016

Pd(II), Arylation [ref. 40];

(2) Ge, 2018

Pd(II), Arylation [ref. 43]

图 1 碳氢键官能团化所用基于亚胺的瞬态导向基(不对称的例子加星号标示)

Figure 1 Imine-based transient directing groups for the functionalization of $\mathrm{C}-\mathrm{H}$ bonds (enantioselective examples are marked with an asterisk)

Breit et al., 2011

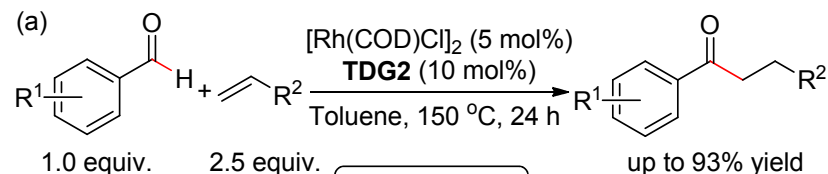

1.0 equiv.

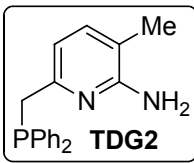

(b)

$\left[\mathrm{Rh}(\mathrm{COD})_{2}\right] \mathrm{BF}_{4}(5 \mathrm{~mol} \%)$ $\underset{\text { Toluene, } 150{ }^{\circ} \mathrm{C}, 1 \mathrm{~h}}{\stackrel{\text { TDG2 }(5 \mathrm{~mol} \%)}{\longrightarrow}}$

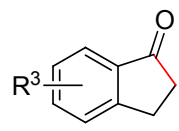

1.0 equiv.

up to $98 \%$ yield

图式 3 铑催化的烯烃分子间和分子内的氢酰化反应

Scheme 3 Rhodium-catalyzed inter- and intra-molecular hydroacylation of alkenes
化剂，还能增强瞬态导向基对催化活性中心铑的结合能 力, 从而增加铑催化剂的有效摩尔浓度, 进一步促进碳 氢键活化.

2012 年, Douglas 课题组 ${ }^{[11]}$ 基于 2 -氨基-3-甲基吡啶 的骨架也发展了新的瞬态导向基 TDG3 和 TDG4, 并成 功实现了铑催化烯烃分子内的氢酰化反应，合成了一系 列六/七元环的环酮 (Scheme 4, a). 当在瞬态导向基中吡 啶环的 5 位取代基引入手性时(TDG4), 能以 31\%的ee 实现对映选择性的氢酰化反应(Scheme 4, b).

\subsection{2 芳基 $\mathrm{C}\left(\mathrm{sp}^{2}\right)$ 一 $\mathrm{H}$ 键活化}

2002 年, Jun 课题组 ${ }^{[12]}$ 报道了一例苄胺(TDG5)作为 瞬态导向基，铑催化芳香酮的邻位 $\mathrm{C}\left(\mathrm{sp}^{2}\right)-\mathrm{H}$ 键的烷基 化反应(Scheme 5, a). 该反应具有很好的单烷基化选择 
Douglas et al., 2012

(a)

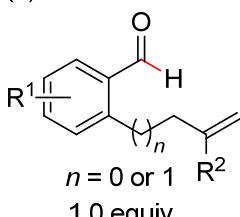

$\left[\mathrm{RhCl}(\mathrm{coe})_{2}\right]_{2}(2.5 \mathrm{~mol} \%)$ $10 \mathrm{~mol} \%$ TDG1 or TDG3 $\mathrm{PhNH}_{2}$ (1.2 equiv.)

$\mathrm{PPh}_{3}(5 \mathrm{~mol} \%)$ $\mathrm{BzOH}(10 \mathrm{~mol} \%)$ $\mathrm{PhCF}_{3}, 100^{\circ} \mathrm{C}$

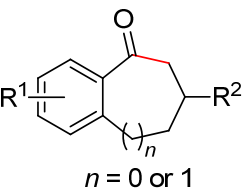

1.0 equiv.

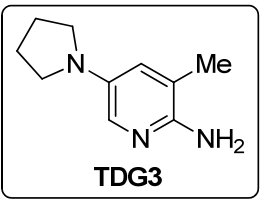

(b)<smiles>C=C(C)CCc1ccccc1C=O</smiles>
$\left[\mathrm{RhCl}(\mathrm{coe})_{2}\right]_{2}(2.5 \mathrm{~mol} \%)$ TDG4 (25 mol\%) $\mathrm{PhNH}_{2}$ (1.2 equiv.)

$\mathrm{PPh}_{3}(5 \mathrm{~mol} \%)$ $\mathrm{BzOH}(10 \mathrm{~mol} \%)$ $\mathrm{PhCF}_{3}, 80^{\circ} \mathrm{C}$ up to $86 \%$ yield<smiles>Cc1cc(N2C(=O)OC(c3ccccc3)(c3ccccc3)C2Cc2ccccc2)cnc1N</smiles>

图式 4 铑催化的分子内氢酰化制备六/七元环的环酮

Scheme 4 Rhodium-catalyzed intramolecular hydroacylation to form six and seven-membered ring ketones

性, 作者认为这可能是因为邻位烷基化的产物的位阻作 用，阻碍了二次碳氢键活化环金属中间体的形成 (Scheme 5, b).

Jun et al., 2002

(a)<smiles>[R]C(=O)c1cc[R1]cc1</smiles>

(1) $5 \mathrm{~mol} \%\left[\mathrm{RhCl}\left(\mathrm{PPh}_{3}\right)_{3}\right]$<smiles>[R]C=C</smiles>
$\underset{(2) \mathrm{H}^{+} / \mathrm{H}_{2} \mathrm{O}}{\stackrel{5}{50 \mathrm{~mol} \% \text { TDG5 }} \text { Toluene, } 150^{\circ} \mathrm{C}, 6 \mathrm{~h}}$ 1.0 equiv.<smiles>NCc1ccccc1CCc1ccccc1</smiles>

(b)<smiles>C/C=C(\C=C(/C)c1ccccc1CCCBr)CCBr</smiles>

图式 5 铑催化芳香酮的邻位 $\mathrm{C}\left(\mathrm{sp}^{2}\right)-\mathrm{H}$ 键的烷基化反应 Scheme 5 Rhodium-catalyzed ortho-C $\left(\mathrm{sp}^{2}\right)-\mathrm{H}$ alkylation of aromatic ketones

2006 年, Takai 课题组 ${ }^{[13]}$ 报道了一例以对甲氧基苯 胺(TDG6)为瞬态导向基, 铼催化芳香酮和 $\alpha, \beta$-不饱和 酯的碳氢键活化环化反应(Scheme 6). 该 $[3+2]$ 环化反 应仅产生水这一副产物, 是一例高效且绿色的合成狮衍
生物的方法.

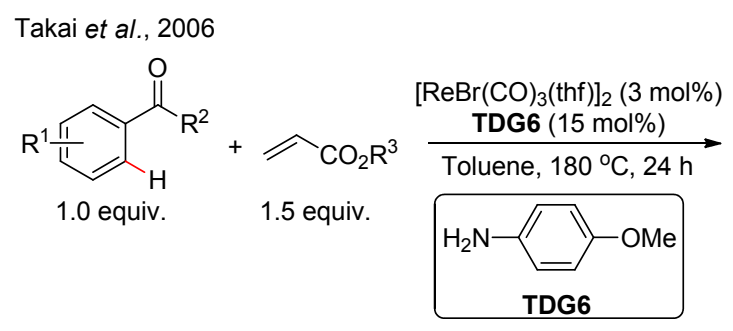<smiles>[R]C(=O)C1=C([R])c2cc[R1]cc2C1</smiles>

up to $93 \%$ yield

图式 6 铼催化芳香酮和 $\alpha, \beta$-不饱和酯的碳氢键活化/环化反 应

Scheme 6 Rhenium-catalyzed annulation of aromatic ketones and $\alpha, \beta$-unsaturated esters through $\mathrm{C}-\mathrm{H}$ bond activation

2013 年, 成江课题组 ${ }^{[14]}$ 报道了一例以酰基肼(TDG7) 为瞬态导向基，铑催化芳香醛与炔烃的环化制备茚酮的 反应(Scheme 7). 同年, Seayad 课题组 ${ }^{[15]}$ 报道了以对三 氟甲基苯胺(TDG8)为瞬态导向基, 铑催化醛的氧化偶 联制备 C(3)-取代的苯并呋喃酤类化合物的反应(Scheme $8)$. 该反应涉及一个串联的邻位碳氢键活化一插入-环化 过程. 2019 年, 汪君课题组 ${ }^{[16]}$ 则以手性脂肪胺 TDG9 为 瞬态导向基, 成功实现铑催化芳香醛的不对称碳氢键活 化合成手性苯并呋喃酮类化合物(Scheme 9).

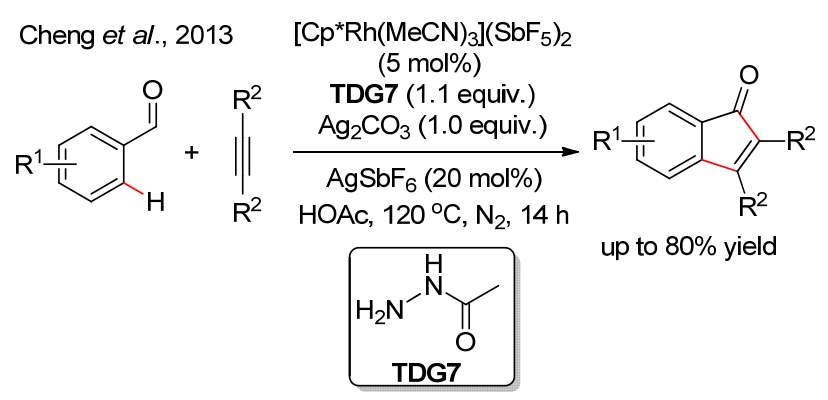

图式 7 铑催化芳香醛和炔烃的直接环化制备狮酮的反应 Scheme 7 Rhodium-catalyzed direct annulation of aromatic aldehydes with alkynes leading to indenones

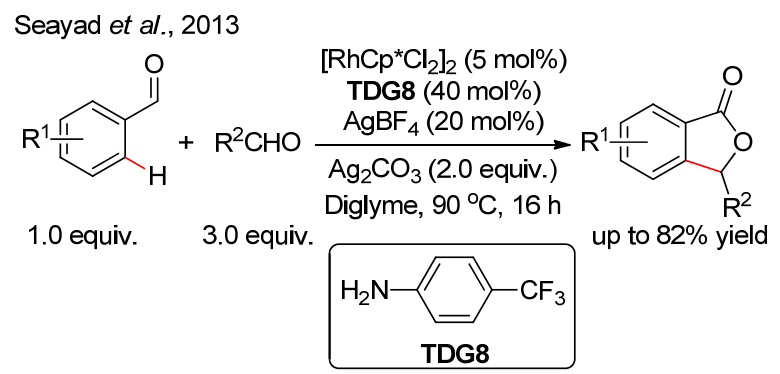

图式 8 铑催化醛的氧化偶联制备苯并呋喃酮类化合物 Scheme 8 Rhodium-catalyzed oxidative coupling of aldehydes to form phthalides 


\section{Wang et al., 2019}

(a) Homocoupling

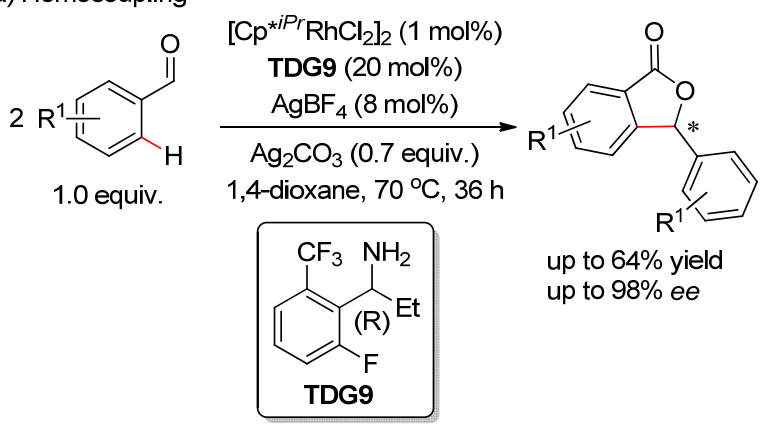

(b) Heterocoupling

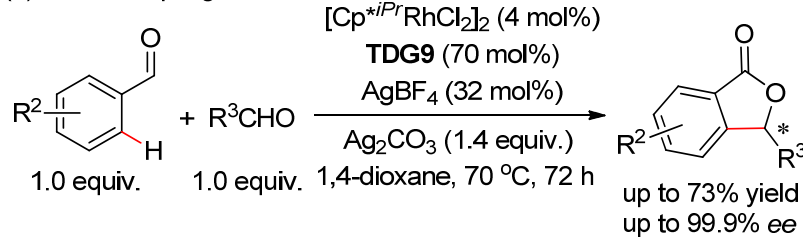

图式 9 铑催化芳香醛的不对称碳氢键活化合成手性苯并呋 喃酮类化合物

Scheme 9 Synthesis of chiral phthalides from aromatic aldehydes via rhodium-catalyzed asymmetric $\mathrm{C}-\mathrm{H}$ activation

最初, 基于瞬态导向策略的碳氢键活化主要集中于 铑(I)催化，其他金属催化的反应尚未得到报道. 2016 年, 余金权课题组 ${ }^{[36]}$ (见 1.1.3 节)开创性地以 $\alpha$-氨基酸作为 瞬态导向基, 实现了钯催化的 $\mathrm{C}\left(\mathrm{sp}^{3}\right)-\mathrm{H}$ 键芳基化反应. 与之前的导向基相比, 氨基酸更加廉价易得, 结构也更 丰富多样, 且可以引入手性中心进行手性诱导, 同时其 羧酸部分配位能力较弱, 利于后续金属的解离再生. 因 此, 基于瞬态导向策略的钯催化碳氢键活化受到广泛关 注, 并在最近几年取得了快速的发展.

2017 年, 余金权课题组 ${ }^{[17]}$ 报道了以 2-甲基丙氨酸 (TDG10)为瞬态导向基, 实现了芳香醛邻位 $\mathrm{C}\left(\mathrm{sp}^{2}\right)-\mathrm{H}$ 键的芳基化反应(Scheme 10). 该反应具有良好的官能 团兼容性，对于间位或邻位取代的芳基碘也能兼容. 值 得一提的是, 当使用无取代的磑代噻吩或碘代吡啶作为 芳基化试剂时，反应几乎不发生，但当这些杂环的 2 位 被取代后就能得到目标产物.

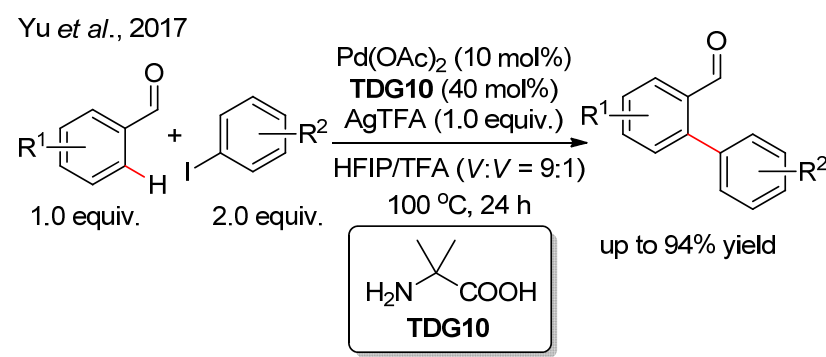

图式 10 钯催化芳香醛的邻位 $\mathrm{C}\left(\mathrm{sp}^{2}\right)-\mathrm{H}$ 键芳基化反应 Scheme 10 Palladium-catalyzed ortho- $\mathrm{C}\left(\mathrm{sp}^{2}\right)-\mathrm{H}$ arylation of aromatic aldehydes
同年, Sorensen 课题组 ${ }^{[18]}$ 报道了钯催化芳香醛邻位 $\mathrm{C}\left(\mathrm{sp}^{2}\right)-\mathrm{H}$ 键的芳基化反应，该反应以廉价易得的邻氨 基苯甲酸(TDG11)作为瞬态导向基，发生芳基醛的邻位 芳基化后，继续对引入芳基的邻位碳氢键进行活化，形 成的跨环环靶中间体对亚胺进行加成/ $\beta$ 氢消除/原位水 解脱除瞬态导向基，最后得到各种取代的芴酮 (Scheme 11). 他们以该反应为关键步骤，经过三步，以 $40 \%$ 的总 收率合成了抗病毒药物 Tilorone.

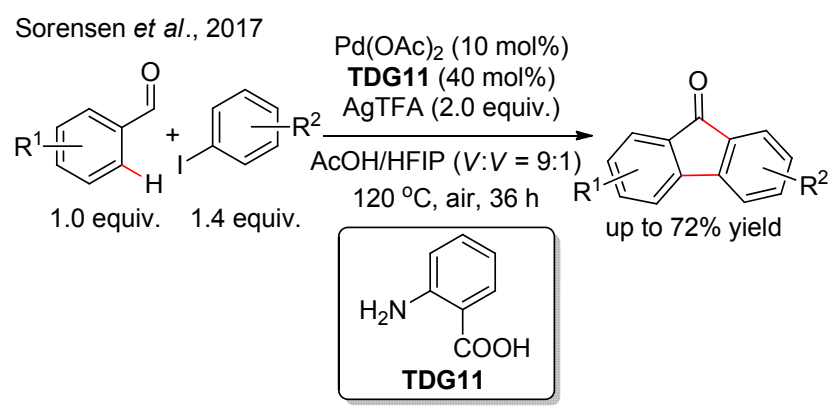

图式 11 由芳香醛和芳基碘经钯催化的双碳氢键活化合成芴 酮

Scheme 11 Synthesis of fluorenones from aromatic aldehydes and aryl iodides via palladium-catalyzed dual $\mathrm{C}-\mathrm{H}$ functionalizations

随后，金钟课题组 ${ }^{[19]}$ 以甘氨酸(TDG12)为瞬态导向 基，成功实现了钯催化芳香酮的邻位 $\mathrm{C}\left(\mathrm{sp}^{2}\right)-\mathrm{H}$ 键的芳 基化反应(Scheme 12). 值得一提的是，作者做了一组平 行实验比较了 $\mathrm{Pd}(\mathrm{OAc})_{2}$ 和 $\mathrm{Pd}\left(\mathrm{PPh}_{3}\right)_{2} \mathrm{Cl}_{2}$ 的催化效果, 实 验发现用 $\mathrm{Pd}\left(\mathrm{PPh}_{3}\right)_{2} \mathrm{Cl}_{2}$ 作催化剂时, 其反应收率明显高 于 $\mathrm{Pd}(\mathrm{OAc})_{2}$. 因此，作者猜测可能膦配体对该邻位 $\mathrm{C}\left(\mathrm{sp}^{2}\right)-\mathrm{H}$ 键的芳基化反应有显著的促进作用，其原因 可能是膦配体通过与 $\mathrm{Pd}(\mathrm{II})$ 的配位稳定了钯催化剂.

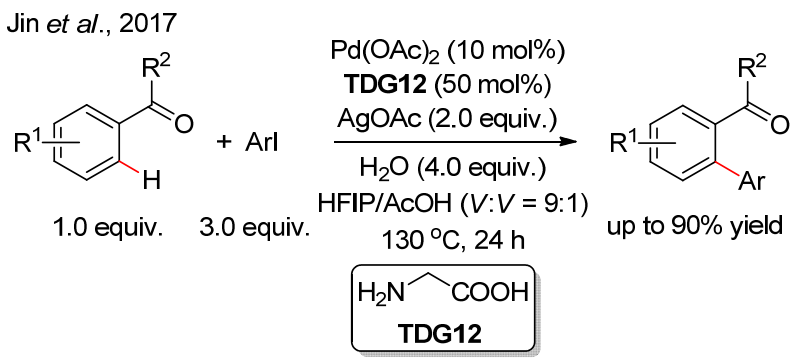

图式 12 钯催化芳香酮的邻位 $\mathrm{C}\left(\mathrm{sp}^{2}\right)-\mathrm{H}$ 键芳基化反应 Scheme 12 Palladium-catalyzed ortho- $\mathrm{C}\left(\mathrm{sp}^{2}\right)-\mathrm{H}$ arylation of aromatic ketones

2017 年, 成江课题组 ${ }^{[20]}$ 报道了以䒾胺(TDG5)为瞬 态导向基，铑催化芳香醛和亚硝基化合物的双边环化制 备非对称唤啶的反应(Scheme 13). 该反应涉及一个串 联的碳氢键胺化一环化一芳构化的过程. 为进一步了解该 反应，作者还做了一些动力学同位素实验：首先，测得 
苯甲醛碳氢键的分子内 $k_{\mathrm{H}} / k_{\mathrm{D}}=2.2$, 亚硝基苯碳氢键的 分子内 $k_{\mathrm{H}} / k_{\mathrm{D}}=1.0$; 然后, 测分子间的动力学同位素效 应, 其结果与分子内的完全一样. 这一结果证明该环化 反应的决速步骤为芳香醛邻位碳氢键的断裂, 而不是芳 香亚硝基化合物的邻位碳氢键的断裂. 实验证明, 在反 应标准条件下羟胺能够被还原成胺, 因此, 作者猜测反 应溶剂二氯乙烷(DCE)在体系中作为还原剂, 起到还原 由碳氢键加成到亚硝基上所生成的羟胺的作用.

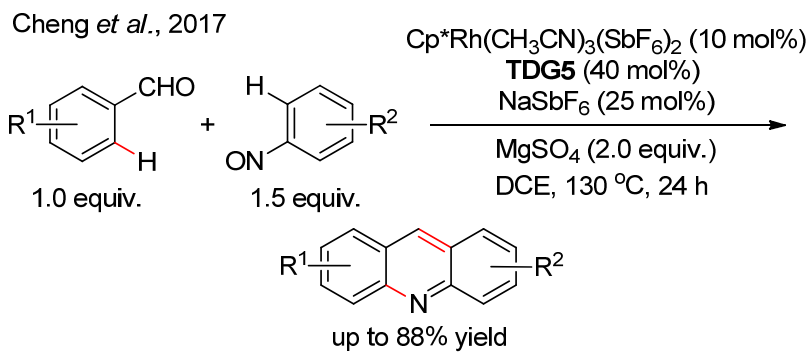

图式 13 铑催化芳香醛与硝基的双边环化制备非对称浐啶 Scheme 13 Rhodium-catalyzed bilateral cyclization of aldehydes with nitrosos toward unsymmetrical acridines

2018 年, 葛海波课题组 ${ }^{[21]}$ 报道了以 3-氨基-3-甲基 丁酸(TDG13)为瞬态导向基, 五元杂芳醛/酮的邻位 $\mathrm{C}\left(\mathrm{sp}^{2}\right)-\mathrm{H}$ 键芳基化反应, 并将该反应应用于发光材料 的合成(Scheme 14).

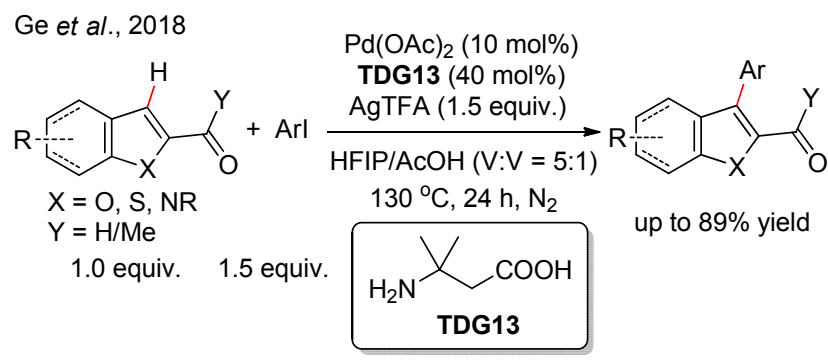

图式 14 钯催化五元杂芳醛/酮的邻位芳基化反应

Scheme 14 Palladium-catalyzed ortho- $\mathrm{C}\left(\mathrm{sp}^{2}\right)-\mathrm{H}$ arylation of five-membered heteroaromatic aldehydes/ketones

2018 年, 王永强课题组 ${ }^{[22]}$ 报道了一例以 2-甲基丙 氨酸(TDG10)为瞬态导向基, 钯催化芳香醛的邻位 $\mathrm{C}\left(\mathrm{sp}^{2}\right)-\mathrm{H}$ 键芳基化反应(Scheme 15). 与之前报道的以 芳基碘为芳基化试剂不同, 该反应直接用芳烃作为芳基

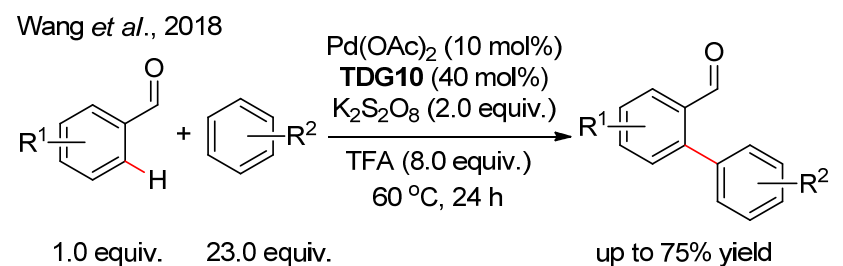

图式 15 钯催化芳香醛与芳烃的直接脱氢芳基化反应

Scheme 15 Palladium-catalyzed direct dehydrogenative arylation of aromatic aldehydes with arenes
化试剂, 具有更高的原子经济性.

2019 年, 张方林课题组 ${ }^{[23]}$ 也报道了钯催化的以间 二(三氟甲基)苯胺(TDG14)为单齿瞬态导向基，芳烃为 芳基化试剂, 两步碳氢键活化构建芴酮衍生物的反应 (Scheme 16).

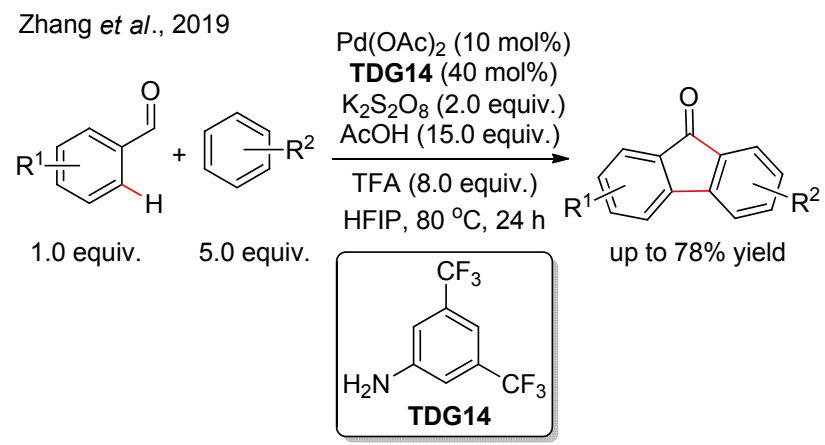

图式 16 由芳香醛和芳烃经钯催化的直接脱氢交叉偶联合成 芴酮

Scheme 16 Synthesis of fluorenones from aromatic aldehydes and arenes via palladium-catalyzed direct dehydrogenative crosscoupling

2018 年, Sorensen 课题组 ${ }^{[24]}$ 以 2-氨基苯磺酸 (TDG15)为瞬态导向基，甲基三氟硼酸钾为甲基化试 剂, 亲电氟代试剂 $(\mathbf{O 1})$ 为氧化剂, 实现了苯甲醛类化合 物邻位 $\mathrm{C}\left(\mathrm{sp}^{2}\right)-\mathrm{H}$ 键甲基化反应(Scheme 17).

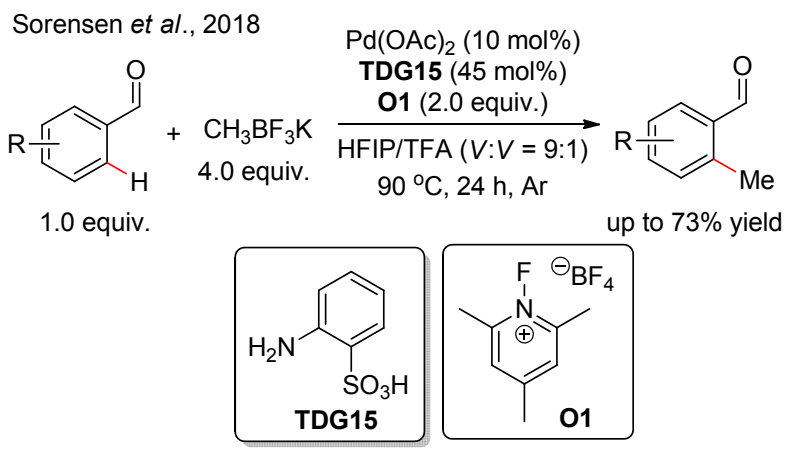

图式 17 钯催化苯甲醛邻位 $\mathrm{C}\left(\mathrm{sp}^{2}\right)-\mathrm{H}$ 键的甲基化反应 Scheme 17 Pd-catalyzed ortho-C( $\left(\mathrm{sp}^{2}\right)-\mathrm{H}$ methylation of benzaldehydes

2018 年, 张方林课题组 ${ }^{[25 \mathrm{a}]}$ 基于瞬态导向策略实现 了钉催化的芳香醛邻位 $\mathrm{C}\left(\mathrm{sp}^{2}\right)-\mathrm{H}$ 键烷基化反应 (Scheme 18). 该反应以2-甲基-3-三氟甲基苯胺(TDG16) 为瞬态导向基, 马来酰亚胺为烷基化试剂 ${ }^{[25 b]}$, 能够兼 容多种官能团. 反应规模可以放大至 $100 \mathrm{mmol}$, 仅需 $0.5 \mathrm{~mol} \%$ 的钉催化剂, 无需柱层析, 即可通过乙酸乙酯 重结晶以 $86 \%$ 的产率得到产物, 充分体现了该反应的高 效性. 烷基化产物还可以通过后期转化制备一系列结构 新颖的螺杂环类化合物. 
Zhang et al., 2018<smiles>[R]N1C(=O)C=CC1=O</smiles>

$\left[\mathrm{Ru}(p \text {-cymene }) \mathrm{Cl}_{2}\right]_{2}(5 \mathrm{~mol} \%)$ TDG16 (20 mol\%) $\mathrm{AgSbF}_{6}(20 \mathrm{~mol} \%)$ 4-chlorobenzoic acid $(50 \mathrm{~mol} \%)$ DCE/HFIP $(V: V=5: 1)$

1.0 equiv. 1.5 equiv. TDG16<smiles>[R][R]1ccc(C2CC(=O)N([R])C2=O)c(C=O)c1</smiles>

up to $90 \%$ yield

图式 18 钉催化的芳香醛的邻位 $\mathrm{C}\left(\mathrm{sp}^{2}\right)-\mathrm{H}$ 键的烷基化反应 Scheme 18 Ruthenium-catalyzed ortho-C( $\left.\mathrm{sp}^{2}\right)-\mathrm{H}$ alkylation of aromatic aldehydes

轴手性联芳化合物是手性配体、催化剂、天然产物 和药物的核心骨架，因此其不对称合成备受关注 ${ }^{[26]}$. 2017 年, 史炳锋课题组 ${ }^{[27 a]}$ 首次报道了基于瞬态导向策 略的钯催化联芳醛的不对称碳氢键烯基化, 高对映选择 性地构建了手性联芳(Scheme 19), 为轴手性化合物提 供了一条全新的合成思路. 该反应以廉价易得的手性 $L-$ (a) Shi et al., 2017

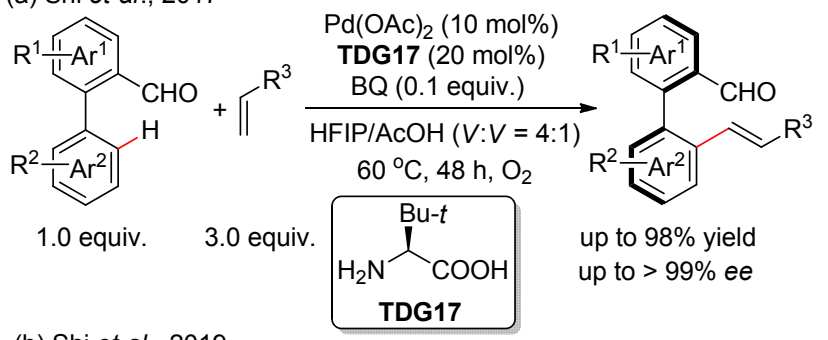

(b) Shi et al., 2019

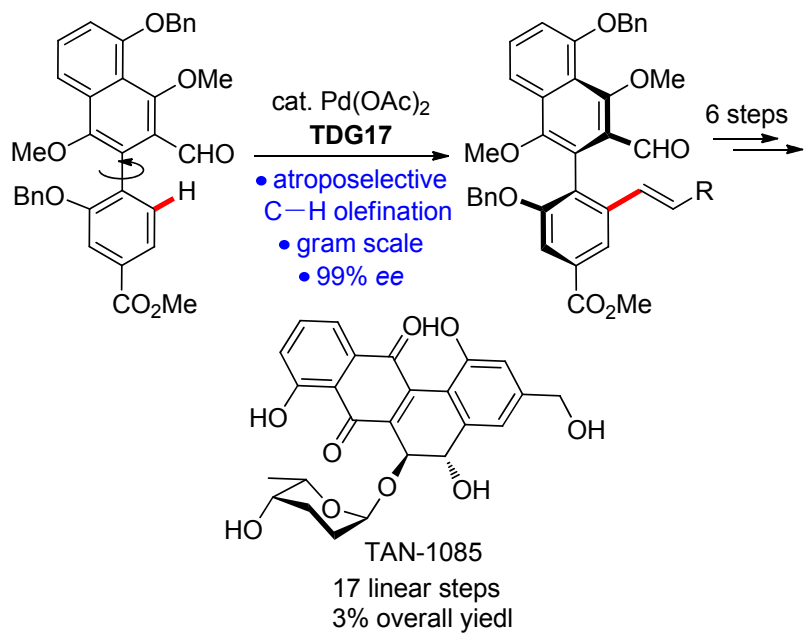

图式 19 钯催化阻转选择性 $\mathrm{C}\left(\mathrm{sp}^{2}\right)-\mathrm{H}$ 键烯基化反应及其在 TAN-1085 合成中的应用

Scheme 19 Palladium-catalyzed atroposelective $\mathrm{C}\left(\mathrm{sp}^{2}\right)-\mathrm{H}$ olefination of biaryl aldehydes and the application in total synthesis of TAN-1085
叔亮氨酸(TDG17)为瞬态导向基，无论是通过动态动力 学拆分(DKR)还是动力学拆分 $(\mathrm{KR})$, 都能以优异的收率 和对映选择性得到轴手性联芳化合物. 以该反应为关键 步骤，史炳锋课题组 ${ }^{[27 b]}$ 也简洁高效地完成了天然产物 TAN-1085 的不对称全合成.

近年来，手性醛作为一类高效的手性有机小分子催 化剂，被广泛应用于有机胺的不对称转化，尤其是联芳 基轴手性醛更是展现出出色的催化活性和手性诱导能 力 $^{[28]}$. 有鉴于此, 史炳锋课题组又将该手性瞬态导向的 不对称碳氢键活化策略拓展到更多的反应类型上，以探 求轴手性联芳化合物的多样性合成及应用. 他们再次以 手性 $L$-叔亮氨酸(TDG17)为瞬态导向基, 先后实现了联

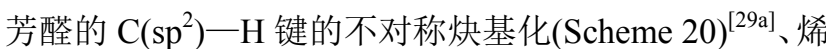
丙基化(Scheme 21) ${ }^{[29 b]}$ 和菜基化(Scheme 22) ${ }^{[30]}$, 各反应 Shi et al., 2018

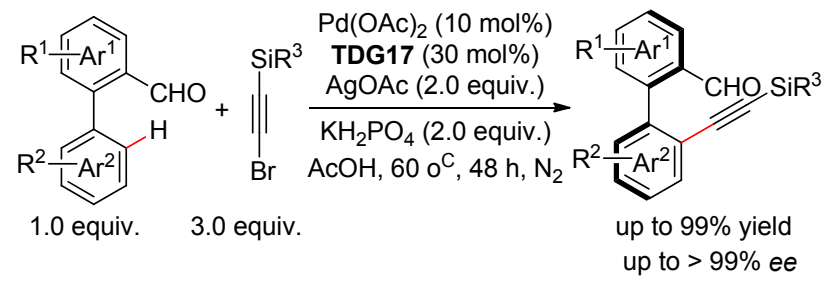

图式 20 钯催化联芳醛类阻转选择性 $\mathrm{C}\left(\mathrm{sp}^{2}\right)-\mathrm{H}$ 键炔基化反 应

Scheme 20 Palladium-catalyzed atroposelective $\mathrm{C}\left(\mathrm{sp}^{2}\right)-\mathrm{H}$ alkynylation of biaryl aldehydes
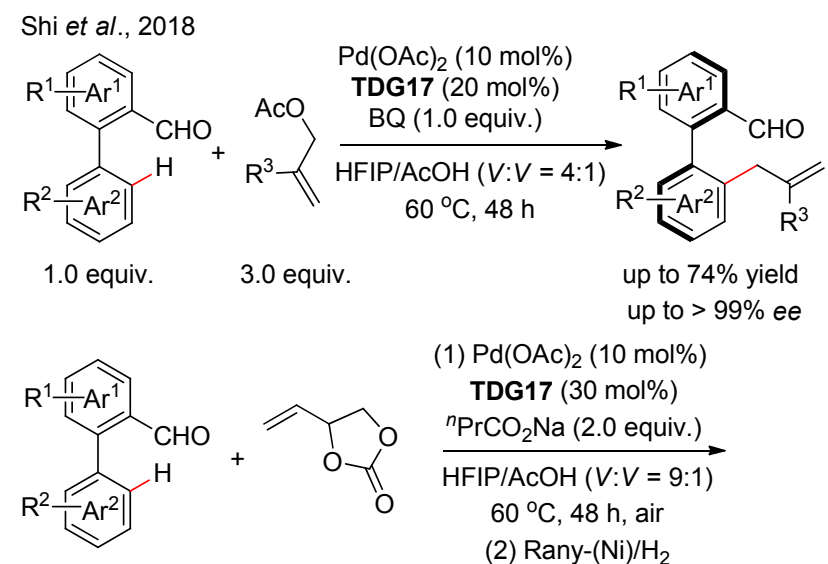

1.0 equiv.

4.0 equiv.

(2) Rany-(Ni)/H

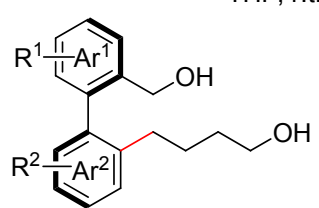

up to $85 \%$ yield up to $>99 \%$ ee

图式 21 钯催化联芳醛类阻转选择性 $\mathrm{C}\left(\mathrm{sp}^{2}\right)-\mathrm{H}$ 键烯丙基化 反应

Scheme 21 Palladium-catalyzed atroposelective $\mathrm{C}\left(\mathrm{sp}^{2}\right)-\mathrm{H}$ allylation of biaryl aldehydes 


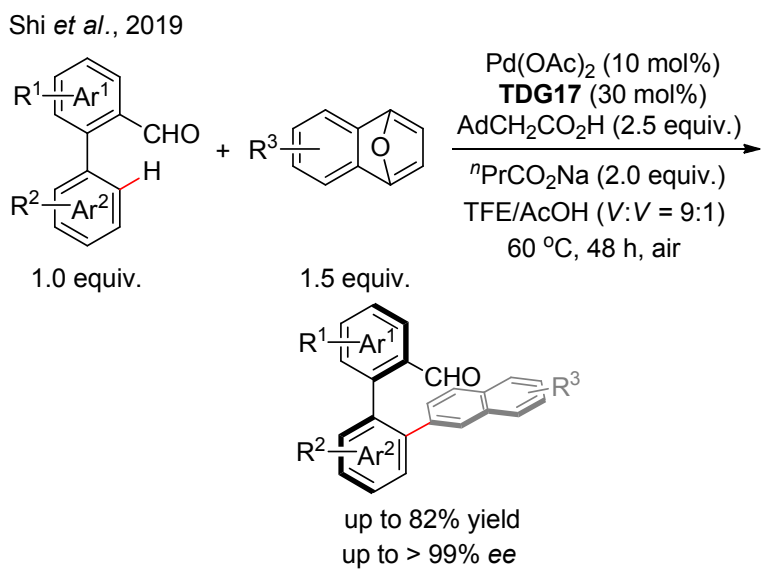

图式 22 钯催化联芳醛类阻转选择性 $\mathrm{C}\left(\mathrm{sp}^{2}\right)-\mathrm{H}$ 键萗基化反 应

Scheme 22 Palladium-catalyzed atroposelective $\mathrm{C}\left(\mathrm{sp}^{2}\right)-\mathrm{H}$ naphthylation of biaryl aldehydes

均能以优异的产率和对映选择性得到轴手性联芳醛化合 物. 以该不对称炔基化反应为关键步骤, 史炳锋课题组 [29a] 也以克级规模, 高立体选择性地实现了天然产物 $(+)$-isoschizandrin 和(+)-steganone 的形式不对称全合成.

含有五元杂芳结构的联芳轴手性化合物具有更低 的翻转能垒, 因此, 其不对称合成更具挑战性 ${ }^{[31]} .2019$ 年, 史炳锋课题组同样发现以手性 $L$-叔亮氨酸(TDG17) 为最合适的瞬态导向基, 能实现翻转能垒相对更小的以 五元杂环为骨架的联芳醛的 $\mathrm{C}\left(\mathrm{sp}^{2}\right)-\mathrm{H}$ 键的不对称炔基

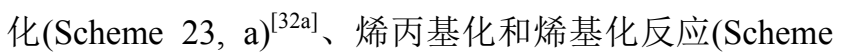
$23, \mathrm{~b})^{[32 b]}$. 谢美华课题组 ${ }^{[32 c]}$ 也报道了一例基于该策略 的 $N$-芳基吲哚阻转选择性的 $\mathrm{C}\left(\mathrm{sp}^{2}\right)-\mathrm{H}$ 键的烯基化反 应. 他们分别以手性 $L$-颉氨酸(TDG18)和 $L$-叔亮氨酸 (TDG17)为瞬态导向基实现该烯基化反应的动态动力 学拆分和动力学拆分 (Scheme 23, c).

尽管不对称碳氢键活化已经被广泛应用于联芳轴 手性化合物的不对称合成, 其在苯乙烯类型的轴手性化 合物的不对称合成中的应用却鲜有报道, 主要挑战在于 该类型阻转手性化合物具有相对较低的翻转能垒, 而碳 氢键活化的发生通常需要相对较高的反应温度 ${ }^{[33]} .2020$ 年, 史炳锋课题组 ${ }^{[34]}$ 又发展了一个由手性 $L$-叔亮氨酸 衍生而来的大位阻的氨基酰胺 TDG19 作为瞬态导向基, 成功实现了丙烯醛类衍生物的阻转选择性的 $\mathrm{C}\left(\mathrm{sp}^{2}\right)-\mathrm{H}$ 键烯基化反应(Scheme 24), 以优异的收率和对映选择 性高效制备了一系列烯烃轴手性化合物. 同时, 作者将 得到的产物经一步简单的转化一醛氧化成卷酸, 得到 对映选择性几乎保持的手性酸, 而后将得到的手性酸作 为手性配体应用于钴催化的硫代酰胺类衍生物的 $\mathrm{C}\left(\mathrm{sp}^{3}\right)-\mathrm{H}$ 键的对映选择性胺化反应. 实验结果表明, 在该反应中这类轴手性苯乙烯系衍生的手性酸的催化 (a) Shi et al., 2019

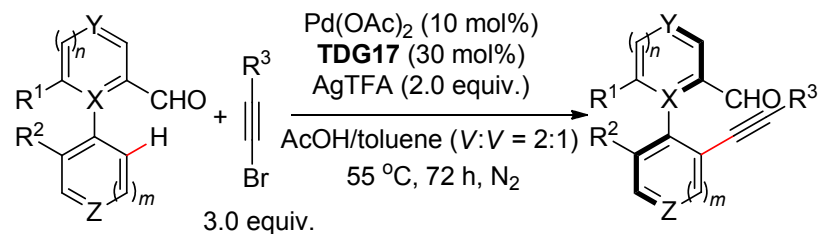

$\mathrm{X}=\mathrm{C}$ or $\mathrm{N}$

$\mathrm{Y}, \mathrm{Z}=\mathrm{C}, \mathrm{S}$ or $\mathrm{O}$

$m, n=0$ or 1

up to $98 \%$ yield

1.0 equiv.

(b) Shi et al., 2019

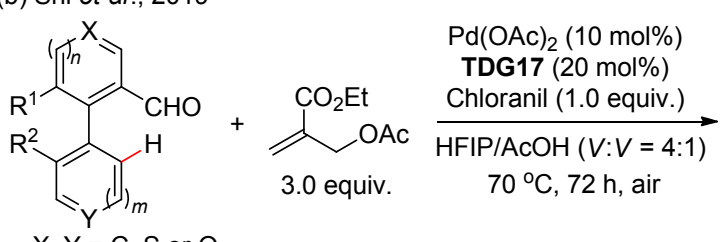

$\mathrm{X}, \mathrm{Y}=\mathrm{C}, \mathrm{S}$ or $\mathrm{O}$ $m, n=0$ or 1

1.0 equiv.

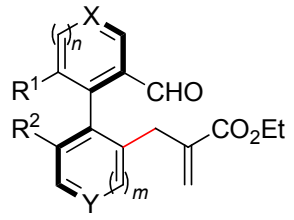

up to $83 \%$ yield

up to $96 \%$ ee

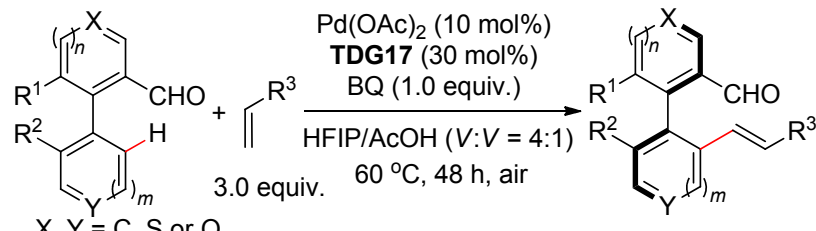

$\mathrm{X}, \mathrm{Y}=\mathrm{C}, \mathrm{S}$ or $\mathrm{O}$

$m, n=0$ or 1

1.0 equiv.

up to $70 \%$ yield up to $99 \%$ ee

(c) Xie et al., 2019

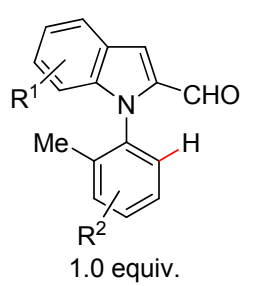

图式 23 钯催化含五元杂环芳香醛的阻转选择性 $\mathrm{C}\left(\mathrm{sp}^{2}\right)-\mathrm{H}$ 键官能团化

Scheme 23 Palladium-catalyzed atroposelective $\mathrm{C}\left(\mathrm{sp}^{2}\right)-\mathrm{H}$ functionalization of pentatomic heteroaromatics

效果明显好于轴手性联芳类型的手性酸. 


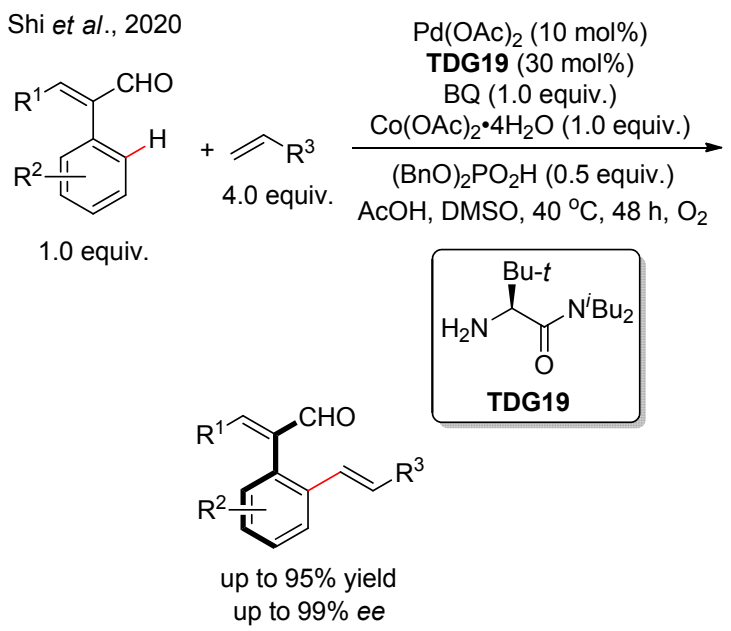

图式 24 钯催化丙烯醉类衍生物阻转选择性 $\mathrm{C}\left(\mathrm{sp}^{2}\right)-\mathrm{H}$ 键烯 基化反应

Scheme 24 Palladium-catalyzed atroposelective $\mathrm{C}\left(\mathrm{sp}^{2}\right)-\mathrm{H}$ olefination of acrylaldehydes

在余金权课题组和史炳锋课题组分别报道了基于 瞬态导向策略实现中心手性和轴手性的构建后, 金钟课 题组 ${ }^{[35]}$ 于 2018 年报道了基于瞬态导向策略构建面手性. 他们同样以手性 $L$-叔亮氨酸(TDG17)为瞬态导向基, 实 现了钯催化的酰基二茂铁的对映选择性 $\mathrm{C}\left(\mathrm{sp}^{2}\right)-\mathrm{H}$ 键芳 基化反应(Scheme 25). 在最初的反应条件的优化过程 中, 他们发现外加的酸的位阻和 $\mathrm{p} K_{\mathrm{a}}$ 均对该反应的活性 和对映选择性有很大的影响. 因此, 他们猜测羧酸所扮 演的角色不仅是能促进亚胺中间体的形成, 还可能参与 了这一对映选择性芳基化的协同金属化一一去质子化 (Concerted Metallation-Deprotonation, CMD)过程. 为证 明该反应的实用性，他们还将芳基化的产物进一步转化 成了基于二茂铁骨架的手性膦配体, 并应用于不对称催 化反应中.

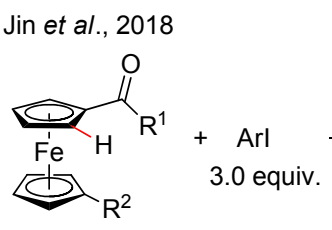

1.0 equiv.

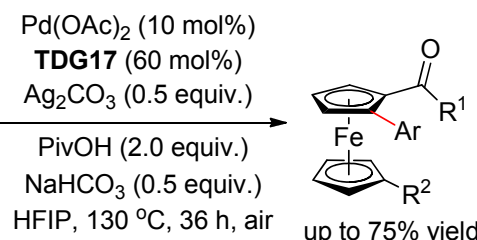

up to $75 \%$ yield up to $98 \%$ ee
图式 25 钯催化酰基二茂铁的不对称 $\mathrm{C}\left(\mathrm{sp}^{2}\right)-\mathrm{H}$ 键芳基化反 应

Scheme 25 Palladium-catalyzed enantioselective $\mathrm{C}\left(\mathrm{sp}^{2}\right)-\mathrm{H}$ arylation of ferrocenyl ketones

\subsection{3 $\mathrm{C}\left(\mathrm{sp}^{3}\right)$ - $\mathrm{H}$ 键活化}

2016 年, 余金权课题组 ${ }^{[36]}$ 首次使用廉价易得的甘 氨酸(TDG12)作为瞬态导向基, 成功地实现了芳香醛的 芐位 $\mathrm{C}\left(\mathrm{sp}^{3}\right)-\mathrm{H}$ 键的芳基化反应(Scheme $26, \mathrm{a}$ ). 该体系 不但能兼容一系列的官能团，如卤原子、硝基、醛基、
酮、酯、羧基等，而且也能兼容杂环芳基碘．他们也成 功地将该体系拓展到了芳香醛的茮位 $\mathrm{C}\left(\mathrm{sp}^{3}\right)-\mathrm{H}$ 键的不 对称芳基化(Scheme 26, b) ${ }^{[36]}$. 通过系统的笁选, 他们发 现当使用手性 $L$-叔亮氨酸(TDG17)作为瞬态导向基时, 能以优异的产率和对映选择性得到芳基化产物。值得一 提的是，在反应优化过程中，他们发现严重的物料不守 恒现象，当反应体系加入水作为添加剂，则可以克服这 一难题. 他们猜测可能是由于亚胺的形成与碳氢键的断 裂这两步反应速率不匹配，从而造成亚胺中间体的分解 消耗，所以加水可以降低反应过程中亚胺中间体的浓度 尽可能阻止其分解.

Yu et al., 2016
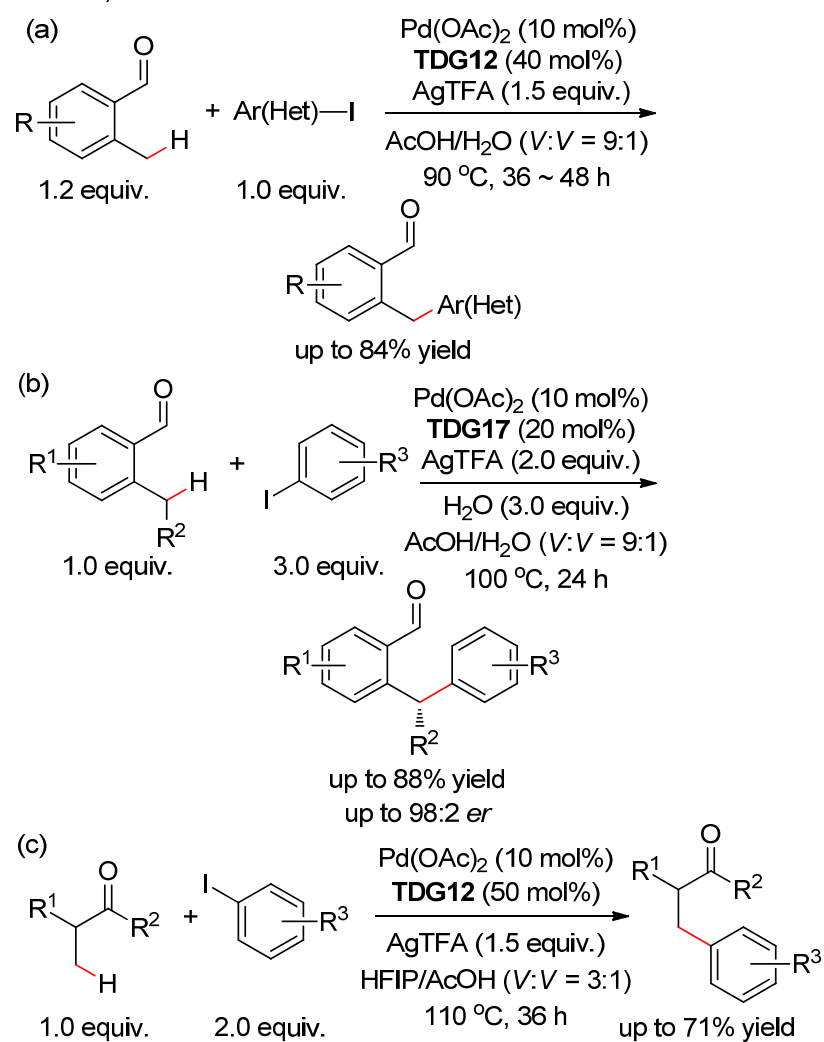

图式 26 钯催化芳香醛苄位 $\mathrm{C}\left(\mathrm{sp}^{3}\right)-\mathrm{H}$ 键芳基化和脂肪酮 $\beta$ - $\mathrm{C}\left(\mathrm{sp}^{3}\right)-\mathrm{H}$ 键芳基化反应

Scheme 26 Palladium-catalyzed benzylic $\mathrm{C}\left(\mathrm{sp}^{3}\right)-\mathrm{H}$ arylation of aromatic aldehydes and $\beta-\mathrm{C}\left(\mathrm{sp}^{3}\right)-\mathrm{H}$ arylation of aliphatic ketones

除此之外，他们 ${ }^{[36]}$ 还将该策略成功拓展到了脂肪 酮底物上. 同样是以甘氨酸(TDG12)为瞬态导向基，能 实现脂肪酮的 $\beta-\mathrm{C}\left(\mathrm{sp}^{3}\right)-\mathrm{H}$ 键的芳基化反应(Scheme 26 , c). 该反应体系不但能兼容带各种给/吸电子基团的芳基 碘，还能活化环状脂肪酮上亚甲基的 $\beta-\mathrm{C}\left(\mathrm{sp}^{3}\right)-\mathrm{H}$ 键， 得到具有很好的非对映选择性的顺式产物. 此外，当 $\beta$ 位没有氢时能活化 $\gamma-\mathrm{C}\left(\mathrm{sp}^{3}\right)-\mathrm{H}$ 键. 但是该工作的 $\beta-\mathrm{C}\left(\mathrm{sp}^{3}\right)-\mathrm{H}$ 键的活化还主要是集中在一级碳氢键上, 
这可能是因为空间位阻的干扰，使得钯插入亚甲基的碳 氢键相对于插入一级碳氢键会更难.

胡立宏课题组 ${ }^{[37 \mathrm{a}]}$ 于 2016 年也报道了一例以乙酰肼 (TDG7)作为瞬态导向基, 钯催化的邻甲基苯甲醛苄位 $\mathrm{C}\left(\mathrm{sp}^{3}\right)-\mathrm{H}$ 键芳基化反应(Scheme 27). 他们发现含有其 他离去基团的芳基化试剂, 如 $\mathrm{Br}, \mathrm{Cl}, \mathrm{TsO}$ 和 $\mathrm{TfO}$, 均不 反应，仅芳基碘适合. 同时芳基碘上的位阻效应对反应 活性影响很大，对位取代的碘苯的反应活性明显大于 邻、间位取代的, 而邻位取代的碘苯几乎不反应. 最近, 包永胜课题组 ${ }^{[37 b]}$ 成功地将该策略应用于纳米钯催化的 非均相体系.

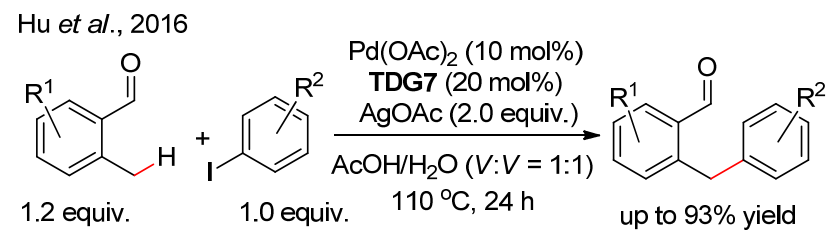

图式 27 钯催化芳香醛苄位 $\mathrm{C}\left(\mathrm{sp}^{3}\right)-\mathrm{H}$ 键芳基化反应 Scheme 27 Palladium-catalyzed benzylic $\mathrm{C}\left(\mathrm{sp}^{3}\right)-\mathrm{H}$ arylation of aromatic aldehydes

2018 年, Jung、Kim 课题组和张方林课题组也分别 报道了同一策略的芳香醛苄位 $\mathrm{C}\left(\mathrm{sp}^{3}\right)-\mathrm{H}$ 键芳基化反应. Jung 和 Kim 课题组 ${ }^{[38]}$ 使用自己设计合成的, 由氨基酸 和氨基醇构成的瞬态导向基 TDG20, 通过反应体系的 调控, 可以分别实现芳基化以及芳基化后关环芳构化合 成葸类化合物 (Scheme 28). 张方林课题组 ${ }^{[39]}$ 则使用甘 Jung and Kim et al., 2018

(a)<smiles>CCc1ccccc1C=O</smiles>
1.2 equiv. $+$<smiles>[R]c1ccc([14OH])cc1</smiles>
$\mathrm{Pd}(\mathrm{TFA})_{2}(10 \mathrm{~mol} \%)$ TDG20 (40 mol\%) AgTFA (1.5 equiv.) $\mathrm{AcOH} / \mathrm{H}_{2} \mathrm{O}(\mathrm{V}: \mathrm{V}=9: 1)$

$90^{\circ} \mathrm{C}, 12 \mathrm{~h} \quad$ up to $91 \%$ yield<smiles>CC(C)(CO)NC(=O)[C@@H](N)CCCO</smiles>

(b)<smiles>O=Cc1ccccc1C=O</smiles>
1.2 equiv.<smiles>Ic1cc[R]cc1</smiles>
1.0 equiv.<smiles>[R]c1ccc2cc3ccccc3cc2c1</smiles>

up to $37 \%$ yield

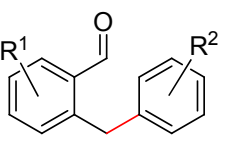

氨酸(TDG12)作为瞬态导向基，实现苄位 $\mathrm{C}\left(\mathrm{sp}^{3}\right)-\mathrm{H}$ 键 芳基化，并且芳基化产物在三氟甲磺酸( $\mathrm{TfOH})$ 的催化 下，经环化芳构化得到了一系列多环芳烃(Scheme 29).
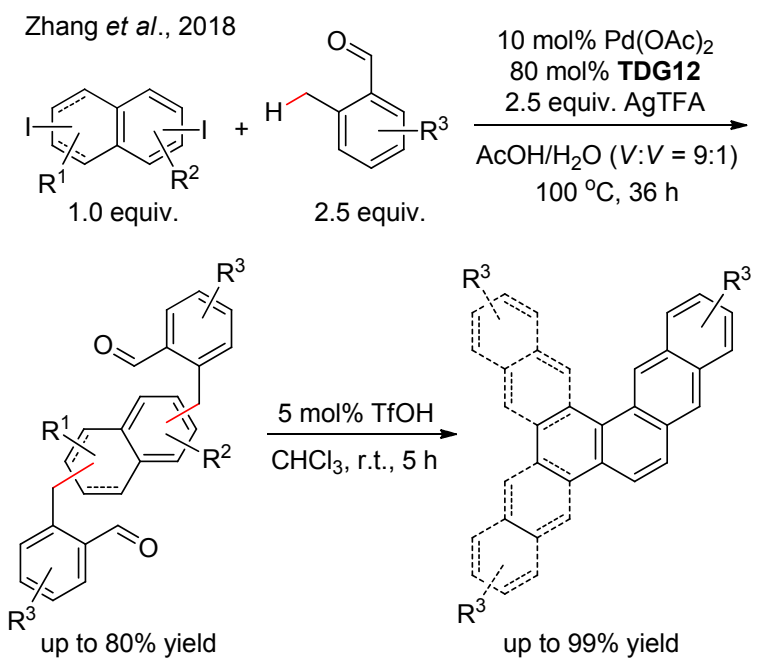

图式 29 基于瞬态导向策略的钯催化多环芳烃的合成 Scheme 29 Palladium-catalyzed synthesis of polycyclic aromatic hydrocarbons (PAHs) via a transient directing group strategy

由于普通脂肪烃 $\mathrm{C}\left(\mathrm{sp}^{3}\right)$ - $\mathrm{H}$ 键相对于芐位 $\mathrm{C}\left(\mathrm{sp}^{3}\right)$ $\mathrm{H}$ 键更加惰性一点, 因此其高效的选择性活化更加困 难. 2016 年, 葛海波课题组 ${ }^{[40]}$ 基于瞬态导向策略成功地 实现了脂肪醛 $\beta-\mathrm{C}\left(\mathrm{sp}^{3}\right)-\mathrm{H}$ 键芳基化反应(Scheme 30). 该反应体系以 $\beta$-丙氨酸(TDG21)作为瞬态导向基，有很 好的官能团兼容性和位点选择性.

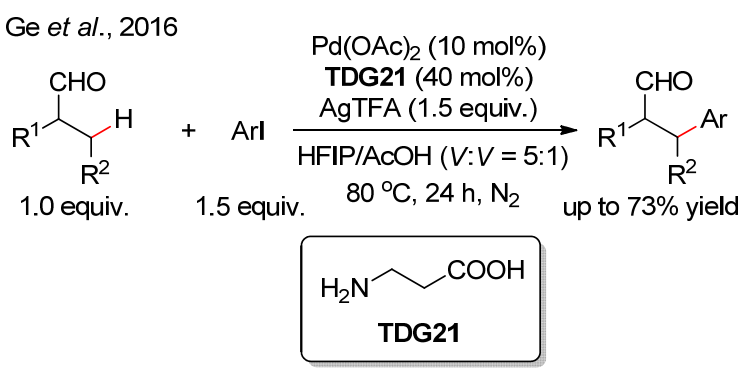

图式 30 钯催化脂肪醛 $\beta-\mathrm{C}\left(\mathrm{sp}^{3}\right)-\mathrm{H}$ 键芳基化反应 Scheme 30 Palladium-catalyzed $\beta-\mathrm{C}\left(\mathrm{sp}^{3}\right)-\mathrm{H}$ arylation of aliphatic aldehydes

次年, Bull 课题组 ${ }^{[41]}$ 基于这一策略也报道了一例脂 肪醛的 $\beta-\mathrm{C}\left(\mathrm{sp}^{3}\right)-\mathrm{H}$ 键的芳基化反应，他们以 $N-(2$-氨基 乙基)-4-甲基苯磺酰胺(TDG22)为瞬态导向基(Scheme 31). 该反应体系仅适用于 $\alpha$ 位季碳中心的脂肪酫，而且 反应的产率中等.

2016 年余金权课题组 ${ }^{[36]}$ 就已报道过一例脂肪酮的 $\beta-\mathrm{C}\left(\mathrm{sp}^{3}\right)-\mathrm{H}$ 键的芳基化反应，但该体系仅适用于一级 碳氢键 (Scheme 26, c). 为解决这一挑战性难题，余金权

图式 28 钯催化 $\mathrm{C}\left(\mathrm{sp}^{3}\right)-\mathrm{H}$ 键芳基化一锅法合成葱类衍生物 Scheme 28 One-pot synthesis of anthracenes by Pd-catalyzed $\mathrm{C}\left(\mathrm{sp}^{3}\right)-\mathrm{H}$ arylation 
Bull et al., 2017

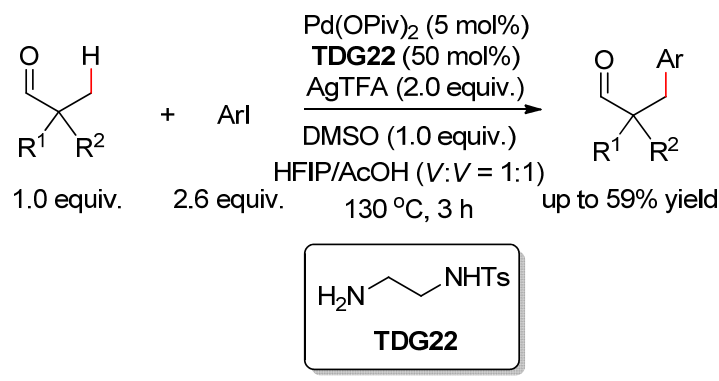

图式 31 钯催化三级醛 $\beta-\mathrm{C}\left(\mathrm{sp}^{3}\right)-\mathrm{H}$ 键芳基化反应

Scheme 31 Palladium-catalyzed $\beta-\mathrm{C}\left(\mathrm{sp}^{3}\right)-\mathrm{H}$ arylation of tertiary aldehydes

课题组 ${ }^{[42]}$ 又发展了一类 $\beta$-氨基酸—— 2 -氨甲基-3-苯基 丙酸(TDG23)作为瞬态导向基, 能够实现钯催化直链脂 肪酮亚甲基 $\beta-\mathrm{C}\left(\mathrm{sp}^{3}\right)-\mathrm{H}$ 键的芳基化反应(Scheme 32). 他们发现 $\beta$-氨基酸与钯催化剂所形成的六元环中间体 对该反应至关重要, 认为这可能有利于促进钯对亚甲基 $\mathrm{C}\left(\mathrm{sp}^{3}\right)-\mathrm{H}$ 键的插入.

$$
\begin{aligned}
& \text { Yu et al., } 2017 \\
& \overbrace{\mathrm{R}^{2}}^{\mathrm{O}}+\mathrm{Arl} \\
& \mathrm{Pd}(\mathrm{OAc})_{2}(10 \mathrm{~mol} \%) \\
& \text { TDG23 (30 mol\%) } \\
& \text { AgTFA (1.0 equiv.) } \\
& \overrightarrow{A g O A c}\left(2.0 \text { equiv.) } R^{1} R_{R^{2}}\right. \\
& 1.0 \text { equiv. } \\
& 2.0 \text { equiv. }
\end{aligned}
$$
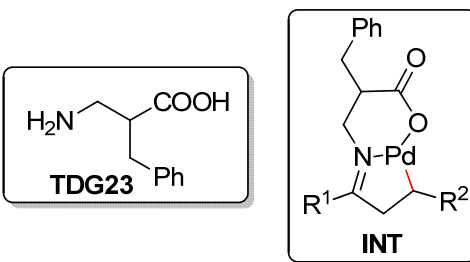

图式 32 钯催化脂肪酮亚甲基 $\beta-\mathrm{C}\left(\mathrm{sp}^{3}\right)-\mathrm{H}$ 键芳基化反应 Scheme 32 Palladium-catalyzed methylene $\beta-\mathrm{C}\left(\mathrm{sp}^{3}\right)-\mathrm{H}$ arylation of aliphatic ketones

2018 年, 葛海波课题组 ${ }^{[43]}$ 同样也用到了一种 $\beta$-氨 基酸—— $\beta$-丙氨酸(TDG21)为瞬态导向基实现了脂肪酮 亚甲基 $\beta-\mathrm{C}\left(\mathrm{sp}^{3}\right)-\mathrm{H}$ 键的芳基化(Scheme 33). 该反应体 系对于直链和环状的脂肪酮都适用, 并具有很好的位点 选择性.

Ge et al., 2018

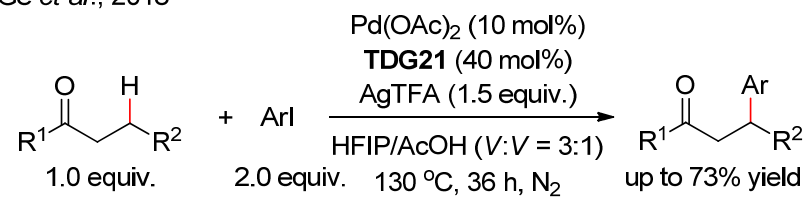

图式 33 钯催化脂肪酮的位点选择性芳基化

Scheme 33 Palladium-catalyzed site-selective arylation of aliphatic ketones

\section{2 碳杂键的构筑}

近年来，基于瞬态导向策略的过渡金属催化碳氢键 活化的反应虽已得到快速发展，但大多都局限在碳一碳 键的构筑，通过该策略实现碳一杂键构筑的例子还不多. 而碳一杂键广泛存在于天然产物分子中，对分子的理化 性质和生物活性等性质均具有重要的影响, 所以通过该 策略实现碳-杂键的构筑具有重大意义.

2016 年, 施章杰课题组 ${ }^{[44]}$ 报道了以间氨基三氟甲 苯 (TDG24) 为瞬态导向基, 铱催化的芳香醛邻位 $\mathrm{C}\left(\mathrm{sp}^{2}\right)-\mathrm{H}$ 键胺化反应(Scheme $\left.34, \mathrm{a}\right)$. 一系列的动力学 实验表明反应中碳一氮键的形成可能是决速步骤. 次年, 何刚课题组 ${ }^{[45]}$ 也报道了以间二(三氟甲基)苯胺(TDG14) 为瞬态导向基, 铱催化的芳香醛邻位 $\mathrm{C}\left(\mathrm{sp}^{2}\right)-\mathrm{H}$ 键胺化 反应(Scheme 34, b).

(a) Shi et al., 2016
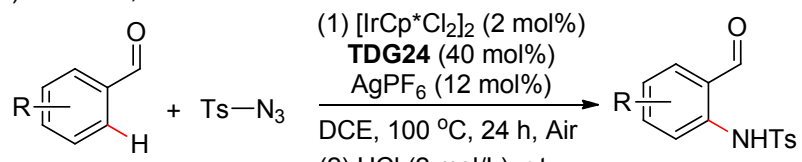

1.0 equiv. 2.0 equiv. (2) $\mathrm{HCl}(2 \mathrm{~mol} / \mathrm{L})$, r.t.

up to $84 \%$ yield

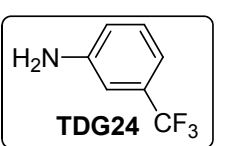

(b) He et al., 2017

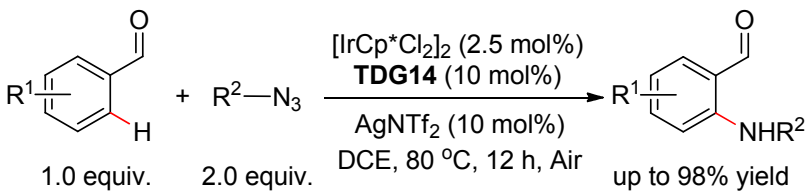

图式 34 铱催化芳香醛邻位 $\mathrm{C}\left(\mathrm{sp}^{2}\right)-\mathrm{H}$ 键胺化反应

Scheme 34 Iridium-catalyzed $\mathrm{C}\left(\mathrm{sp}^{2}\right)-\mathrm{H}$ amidation of aromatic aldehydes

2018 年, 焦宁课题组 ${ }^{[46]}$ 以对三氟甲基苯胺(TDG8) 为瞬态导向基, 成功实现了铑催化芳香醛邻位 $\mathrm{C}\left(\mathrm{sp}^{2}\right)$ $\mathrm{H}$ 键胺化反应(Scheme 35). 同年, Rasheed 课题组 ${ }^{[47]}$ 也报 道了一例基于瞬态策略的钉催化芳香醛邻位 $\mathrm{C}\left(\mathrm{sp}^{2}\right)-\mathrm{H}$ 键胺化反应，无论是 2-甲基-3-三氟甲基苯胺(TDG16)还 是 2-氨基-4-三氟甲基苯甲酸(TDG25)作为瞬态导向基, 均能给出良好的产率(Scheme 36).
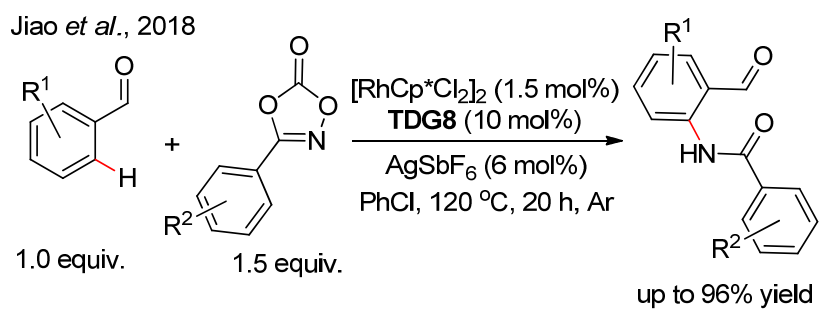

图式 35 铑催化芳香醛邻位 $\mathrm{C}\left(\mathrm{sp}^{2}\right)-\mathrm{H}$ 键胺化反应 Scheme 35 Rhodium-catalyzed $\mathrm{C}\left(\mathrm{sp}^{2}\right)-\mathrm{H}$ amidation of aromatic aldehydes 
Rasheed et al., 2018
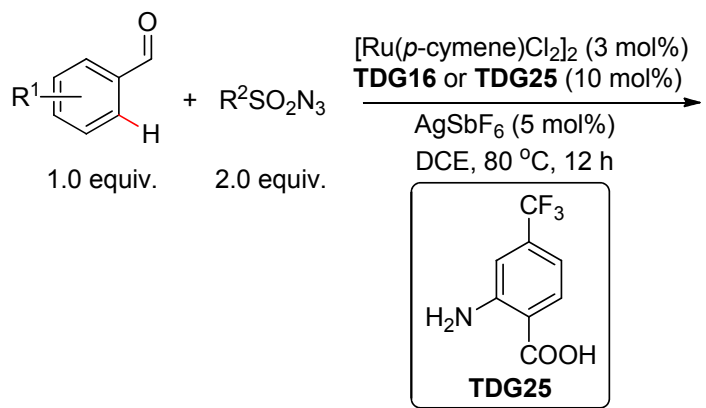<smiles>[R]OS(=O)Nc1cc[R1]([R])cc1C=O</smiles>

up to $96 \%$ yield

图式 36 钉催化芳香醛邻位 $\mathrm{C}\left(\mathrm{sp}^{2}\right)-\mathrm{H}$ 键胺化反应 Scheme 36 Ruthenium-catalyzed $\mathrm{C}\left(\mathrm{sp}^{2}\right)-\mathrm{H}$ amidation of aromatic aldehydes

2019 年, 伍新燕课题组 ${ }^{[48]}$ 报道了以对氯苯胺 (TDG26)为瞬态导向基, 钴催化的芳香醛邻位 $\mathrm{C}\left(\mathrm{sp}^{2}\right)$ $\mathrm{H}$ 键胺化反应(Scheme 37). 他们以该反应为关键步骤, 实现了 $\mathrm{C} 1 \mathrm{r}$ 丝氨酸蛋白酶抑制剂 $\mathbf{I}$ 和弹性蛋白酶抑制剂 II 的合成.

Wu et al., 2019

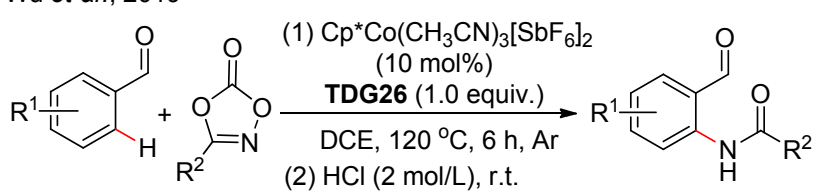
(2) $\mathrm{HCl}(2 \mathrm{~mol} / \mathrm{L})$, r.t.

1.0 equiv. $\quad 2.0$ equiv.

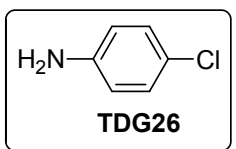

up to $88 \%$ yield

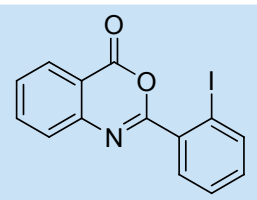

C1r serine protease inhibitor I

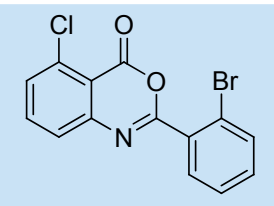

Elastase inhibitor II
图式 37 钴催化芳香醛邻位 $\mathrm{C}\left(\mathrm{sp}^{2}\right)-\mathrm{H}$ 键胺化反应 Scheme 37 Cobalt-catalyzed $\mathrm{C}\left(\mathrm{sp}^{2}\right)-\mathrm{H}$ amidation of aromatic aldehydes

2017 年，余金权课题组 ${ }^{[17]}$ 基于瞬态导向策略实现 了碳-氮键和碳-甾键的构筑. 他们以 2-氟-5-三氟甲基 苯胺(TDG27)为瞬态导向基时实现了铱催化的芳香醛 邻位 $\mathrm{C}\left(\mathrm{sp}^{2}\right)-\mathrm{H}$ 键胺化反应(Scheme $38, \mathrm{a}$ ); 以邻氨基苯 甲酸(TDG11)为瞬态导向基, 则成功实现了钯催化的芳 香醛邻位 $\mathrm{C}\left(\mathrm{sp}^{2}\right)-\mathrm{H}$ 键氯代反应 (Scheme $\left.38, \mathrm{~b}\right)^{[17]}$; 而当 以 2-氨基-4-硝基苯甲酸(TDG28)为瞬态导向基时，则成
功实现了钯催化的芳香醛邻位 $\mathrm{C}\left(\mathrm{sp}^{2}\right)-\mathrm{H}$ 键溴代反应 (Scheme $38, \mathrm{c})^{[17]}$. 他们也成功地将该反应应用于药物 分子的后期修饰上, 得到了一系列胺化、氯代和溴代的 类似物.

Yu et al., 2017 (a)

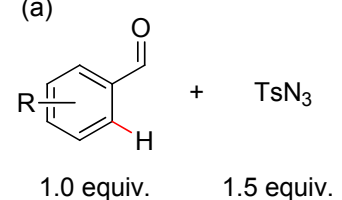

1.0 equiv.

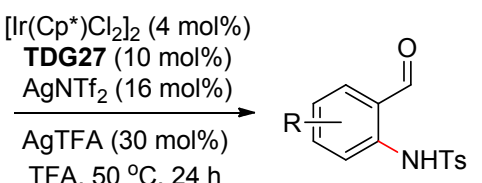

up to $93 \%$ yield

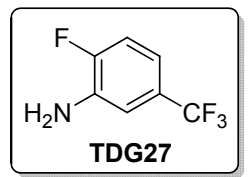

(b)<smiles>O=Cc1cc#[R]cc1</smiles>

$\mathrm{Pd}(\mathrm{OAc})_{2}(10 \mathrm{~mol} \%)$ TDG11 (30 mol\%) $\stackrel{\operatorname{AgTFA}(0.1 \text { equiv.) }}{\longrightarrow}$ TFA (10.0 equiv.) DCE, $60^{\circ} \mathrm{C}, 24 \mathrm{~h}$

1.0 equiv. $\quad 1.5$ equiv.

(c)<smiles>O=Cc1cc[R]cc1</smiles>

$\mathrm{Pd}(\mathrm{OAc})_{2}(10 \mathrm{~mol} \%)$ TDG28 $(50 \mathrm{~mol} \%)$

AgTFA ( 0.1 equiv.)

$p$ - $\mathrm{TsOH}(0.5$ equiv.) DCE/TFA $(V: V=1: 1)$

1.0 equiv.

1.5 equiv. $90^{\circ} \mathrm{C}, 24 \mathrm{~h} \quad$ up to $96 \%$ yield<smiles>Nc1cc([N+](=O)[O-])ccc1C(=O)O</smiles>

图式 38 过渡金属催化芳香醛邻位 $\mathrm{C}\left(\mathrm{sp}^{2}\right)-\mathrm{H}$ 键胺化/氯代/溴 代反应

Scheme 38 Transition-metal-catalyzed $\mathrm{C}\left(\mathrm{sp}^{2}\right)-\mathrm{H}$ amidation/ chlorination/bromination of aromatic aldehydes

2018 年, 该课题组 ${ }^{[49]}$ 也成功地以手性氨基酰胺 (TDG29)为瞬态导向基, 实现了钯催化的芳香醛苠位 $\mathrm{C}\left(\mathrm{sp}^{3}\right)-\mathrm{H}$ 键的不对称氟化(Scheme 39). 实验表明，当 以氨基酸为瞬态导向基时，主要通过分子间 $\mathrm{S}_{\mathrm{N}} 2$ 类型的 $\mathrm{C}-\mathrm{O}$ 键还原消除得到乙酰氧化的产物; 而当采用手性 氨基酰胺为瞬态导向基时, 则主要通过分子内 $\mathrm{C}-\mathrm{F}$ 键 还原消除，得到氟代产物. 他们猜想选择性的来源在于 氨基酰胺类型瞬态导向基团有利于阳离子 $\operatorname{Pd}(\mathrm{IV})$ 中间 体的形成，从而促进 $\mathrm{C}-\mathrm{F}$ 键的还原消除. 同时，他们也 发现大位阻的手性氨基酰胺也更加有利于不对称控制 和促进 $\mathrm{C}-\mathrm{F}$ 键的还原消除. 与醋酸相比, 以缺电子五 氟苯甲酸作为添加剂, 也有利于抑制竞争的 $\mathrm{C}-\mathrm{O}$ 键还 原消除，因为其具有相对更弱的亲核性. 


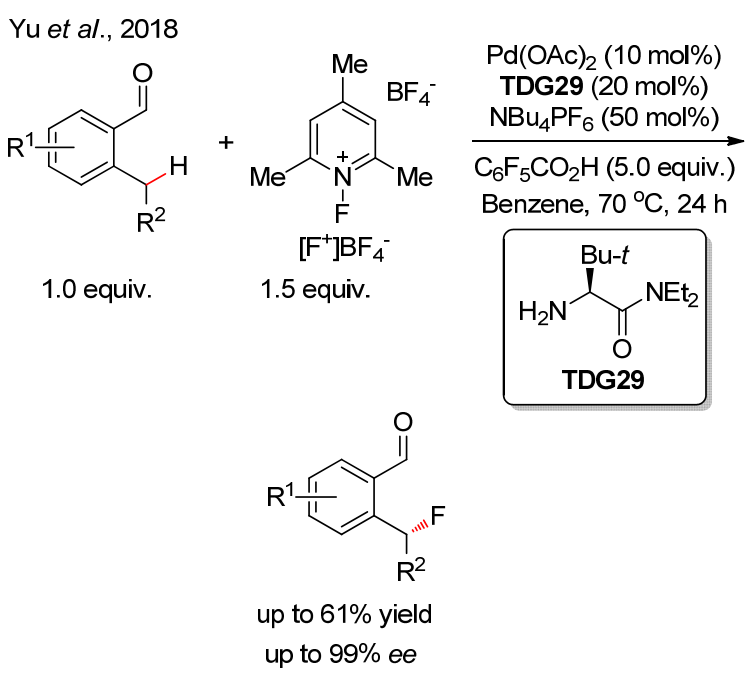

图式 39 钯催化芳香醛苄位 $\mathrm{C}\left(\mathrm{sp}^{3}\right)-\mathrm{H}$ 键不对称氟化

Scheme 39 Palladium-catalyzed benzylic enantioselective $\mathrm{C}\left(\mathrm{sp}^{3}\right)-\mathrm{H}$ fluorination of aromatic aldehydes

Sorensen 课题组 ${ }^{[24]}$ 同样于 2018 年报道了以苯胺2,5-二磺酸(TDG30)为瞬态导向基, 钯催化的芳香醛邻 位 $\mathrm{C}\left(\mathrm{sp}^{2}\right)-\mathrm{H}$ 键氟化反应(Scheme 40$)$. 该课题组 ${ }^{[50]}$ 在此 之前曾报道了基于瞬态导向策略, 钯催化的芳香醛邻位 $\mathrm{C}\left(\mathrm{sp}^{2}\right)-\mathrm{H}$ 键的氧化和氯代反应(Scheme 41). 当以 2-氨 基-4-氯苯甲酸(TDG31)为瞬态导向基, 以对甲苯磺酸为 添加剂，能够实现芳香醛邻位 $\mathrm{C}\left(\mathrm{sp}^{2}\right)-\mathrm{H}$ 键的羟基化 (Scheme 41,a); 当以 2-甲基丙氨酸(TDG10)为瞬态导向 基, 六氟异丙醇(HFIP)为偶联试剂及溶剂时, 则可以实 现多氟烷氧基化反应(Scheme 41, b); 而当以 2-氨基-4氯苯甲酸(TDG31)为瞬态导向基，二氯乙烷(DCE)为氯 源及溶剂时, 则可以得到氯代的产物(Scheme 41, c). 虽 然迄今为止很难通过其他阴离子来捕获 $\mathrm{Pd}(\mathrm{IV})$ 中间体, 但他们的结果很好地证明了外部的亲核试剂对促进由 $\mathrm{F}^{+}$类氧化剂氧化而来的 $\mathrm{Pd}(\mathrm{IV})$ 中间体的还原消除至关 重要.

\section{Sorensen et al., 2018}

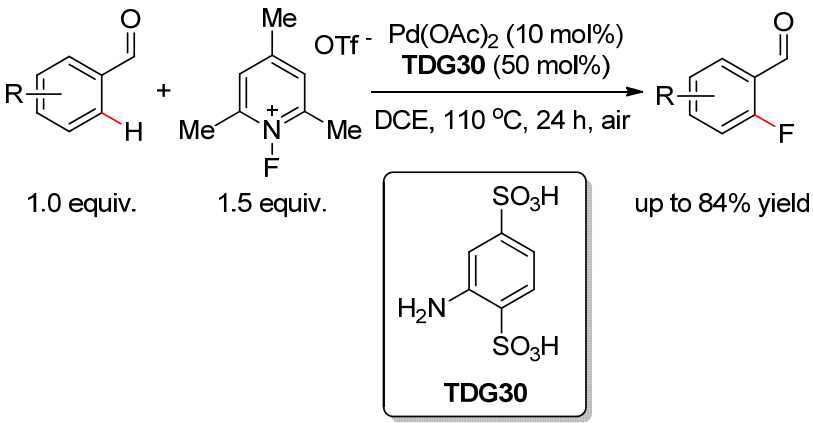

图式 40 钯催化芳香醛邻位 $\mathrm{C}\left(\mathrm{sp}^{2}\right)-\mathrm{H}$ 键氟化反应

Scheme 40 Palladium-catalyzed ortho- $\mathrm{C}\left(\mathrm{sp}^{2}\right)-\mathrm{H}$ fluorination of aromatic aldehydes
Sorensen et al. 2017

(a)

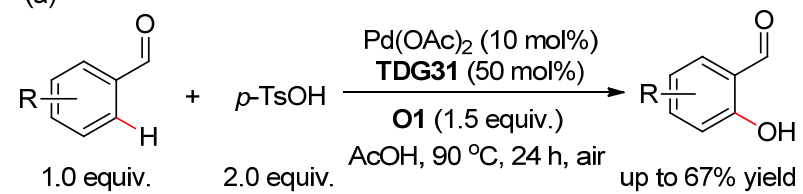

(b)

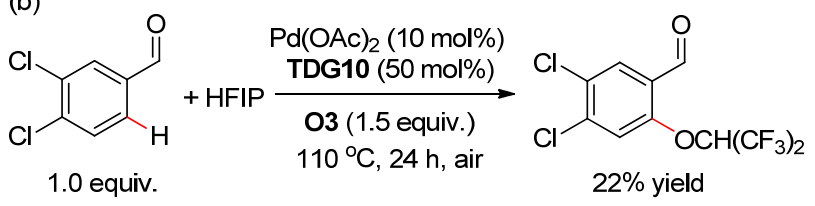

(c)
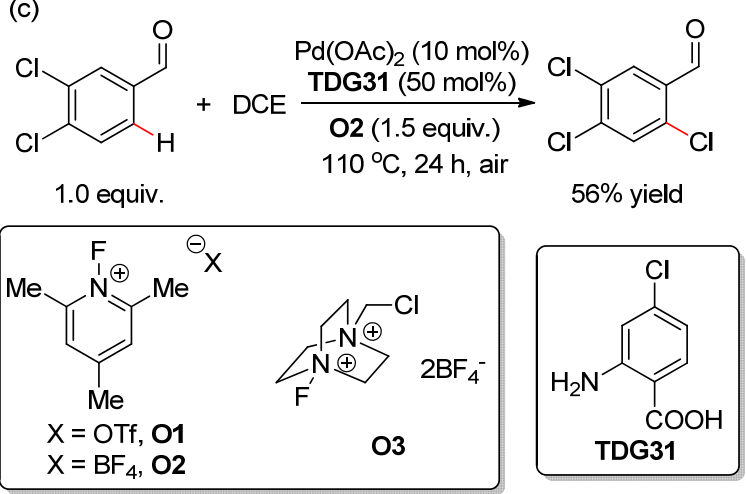

图式 41 钯催化芳香醛邻位 $\mathrm{C}\left(\mathrm{sp}^{2}\right)-\mathrm{H}$ 键羟基化、多氟烷氧 基化和氯代反应

Scheme 41 Palladium-catalyzed ortho-C( $\left(\mathrm{sp}^{2}\right)-\mathrm{H}$ hydroxylation, polyfluoroalkoxylation and chlorination of aromatic aldehydes

2019 年, 张方林课题组 ${ }^{[51]}$ 报道了以单齿瞬态导向 基对三氟甲基苯胺(TDG8)和间氨基三氟甲苯(TDG24) 分别实现芳香醛邻位 $\mathrm{C}\left(\mathrm{sp}^{2}\right)-\mathrm{H}$ 键氯代(Scheme $\left.42, a\right)$ 和 甲氧基化(Scheme 42, b). 他们成功得到了环钯中间体， 并通过单晶衍射确认其结构是由吡啶酮配体桥连的双 核环钯中间体，这一结果证明了在该单齿瞬态导向体系 中, 外加的吡啶酮配体参与了钯催化碳氢键活化. 同年,

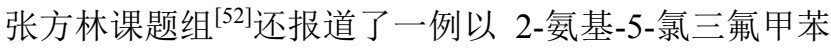
(TDG32)为瞬态导向基, 钯催化的芳香醛邻位 $\mathrm{C}\left(\mathrm{sp}^{2}\right)-$ $\mathrm{H}$ 键溴代反应(Scheme 42, c).

最近, 张方林课题组 ${ }^{[53]}$ 以联苯胺(TDG33)为瞬态导 向基，实现了钯催化芳香醛邻位 $\mathrm{C}\left(\mathrm{sp}^{2}\right)-\mathrm{H}$ 键硒基化反 应(Scheme 43). 该反应底物范围广泛，具有良好的官能 团兼容性，提供了一种高效合成含硒芳香醛的新方法.

史炳锋课题组 ${ }^{[54]}$ 以 3-氨基-3-苯基丙酸(TDG34)为 瞬态导向基，实现了钯催化的芳香醛邻位 $\mathrm{C}\left(\mathrm{sp}^{2}\right)-\mathrm{H}$ 键 硅基化反应(Scheme 44). 值得一提的是，硅基是一类非 常低毒、稳定且有应用价值的基团，能进行多样性的转 化，如氯代、溴代、碘代和偶联等.他们也成功地将该 硅基化的方法应用于手性底物上，在反应过程中，手性 联芳醛的手性能够很好地保持. 
Zhang et al., 2019

(a)<smiles>O=Cc1cc[R]#cc1[CH+]Cl</smiles>

$\mathrm{Pd}(\mathrm{OAc})_{2}(5 \mathrm{~mol} \%)$

TDG8 (10 $\mathrm{mol} \%)$

$\underset{\text { 2-Hydroxy-5-nitro-3-(trifluoro- }}{\stackrel{\text { TFA }(10.0 \text { equiv. })}{\text { methyl)pyridine }(30 \text { mol\%) }} \longrightarrow}$

1.0 equiv. $\quad 1.5$ equiv.

up to $95 \%$ yield

(b)<smiles>O=Cc1cc#[R]cc1</smiles>
1.0 equiv. $\quad 20.0$ equiv.

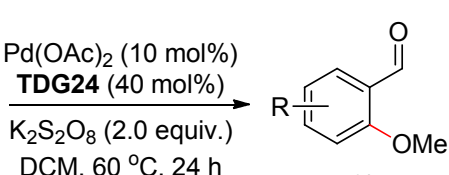
$\mathrm{DCM}, 60^{\circ} \mathrm{C}, 24 \mathrm{~h}$

up to $75 \%$ yield

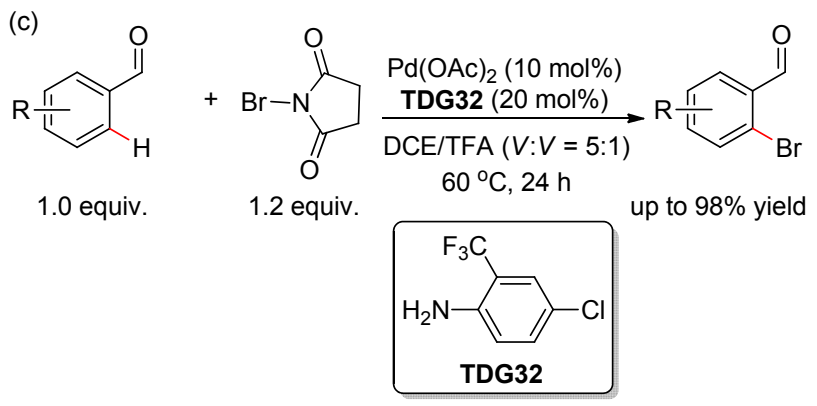

图式 42 钯催化芳香醛邻位 $\mathrm{C}\left(\mathrm{sp}^{2}\right)-\mathrm{H}$ 键氯代、甲氧基化和 溴代反应

Scheme 42 Palladium-catalyzed ortho-C $\left(\mathrm{sp}^{2}\right)-\mathrm{H}$ chlorination, methoxylation and bromination of aromatic aldehydes

Zhang et al., 2019
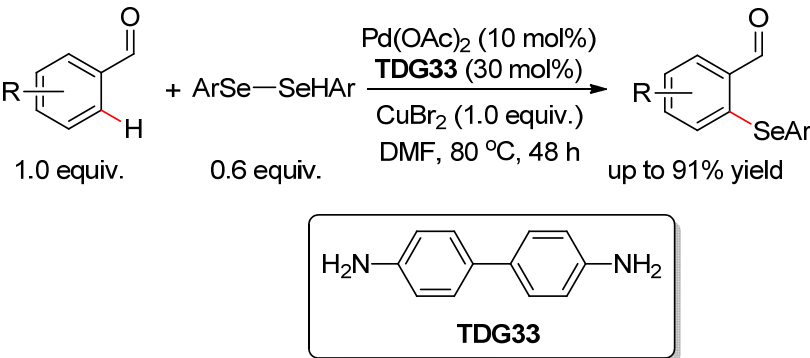

图式 43 钯催化芳香醛邻位 $\mathrm{C}\left(\mathrm{sp}^{2}\right)-\mathrm{H}$ 键硒基化反应

Scheme 43 Palladium-catalyzed ortho- $\mathrm{C}\left(\mathrm{sp}^{2}\right)-\mathrm{H}$ selenylation of aromatic aldehydes

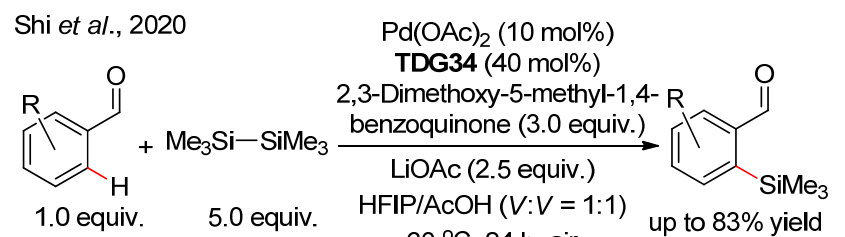

$$
\overbrace{\text { TDG34 }}^{\mathrm{Ch}} \mathrm{COH}
$$

图式 44 钯催化芳香醛邻位 $\mathrm{C}\left(\mathrm{sp}^{2}\right)-\mathrm{H}$ 键硅基化反应 Scheme 44 Palladium-catalyzed ortho- $\mathrm{C}\left(\mathrm{sp}^{2}\right)-\mathrm{H}$ silylation of aromatic aldehydes

\section{2 胺类化合物的碳氢键官能团化}

胺类化合物是一类重要的有机化合物, 胺这一结构 单元广泛存在于天然产物和药物分子中，因此实现胺类 化合物的合成与修饰具有重大意义. 但目前以醛/酮为 瞬态导向基的过渡金属催化胺类衍生物的碳氢键活化 的研究还很少.

2016 年, 葛海波课题组 ${ }^{[5]}$ 以水合乙醛酸(TDG35)为 瞬态导向基, 实现了钯催化脂肪伯胺 $\gamma-\mathrm{C}\left(\mathrm{sp}^{3}\right)-\mathrm{H}$ 键芳 基化(Scheme 45). 由于更容易形成动力学有利的五元 环靶中间体，所有反应底物中均没有观察到 $\beta$-或 $\delta$-位芳 基化的产物 ${ }^{[56]}$.

$$
\begin{gathered}
\text { Ge et al., } 2016 \\
1.0 \text { equiv. } \\
1.5 \text { equiv. }
\end{gathered}
$$

图式 45 钯催化脂肪伯胺 $\gamma-\mathrm{C}\left(\mathrm{sp}^{3}\right)-\mathrm{H}$ 键芳基化反应 Scheme 45 Palladium-catalyzed $\gamma-\mathrm{C}\left(\mathrm{sp}^{3}\right)-\mathrm{H}$ arylation of primary aliphatic amines

同年, 董广涁课题组 ${ }^{[57]}$ 将他们之前就已成功发展 的exo-导向基喹啉-8-甲醛(TDG36)拓展到瞬态导向领 域, 成功实现了钯催化的烷基伯胺和苯胺类衍生物的 $\gamma$ 和 $\delta-\mathrm{C}\left(\mathrm{sp}^{3}\right)-\mathrm{H}$ 键的芳基化反应(Scheme 46).

Dong et al., 2016

(a)

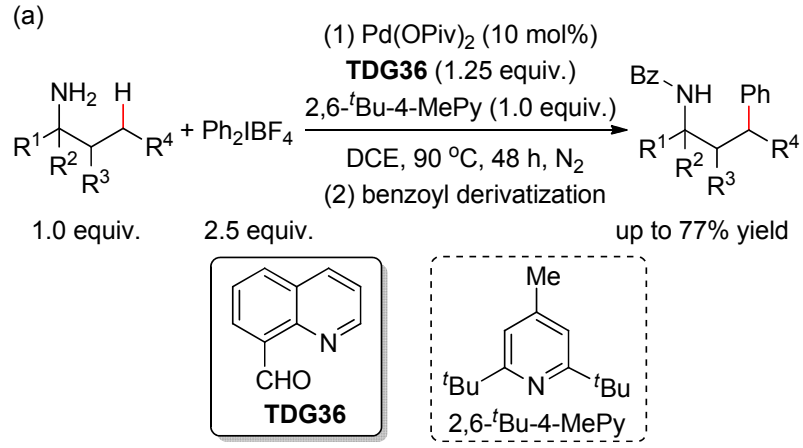

(b)

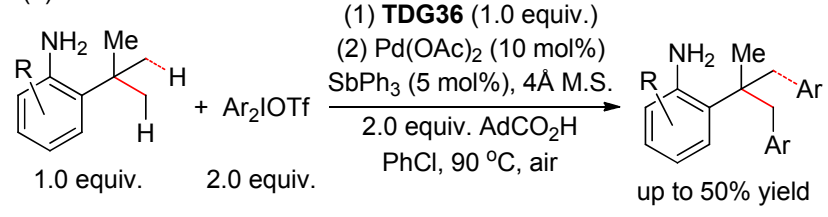

图式 46 钯催化伯胺 $\gamma$-和 $\delta$ - $\mathrm{C}\left(\mathrm{sp}^{3}\right)-\mathrm{H}$ 键芳基化反应 Scheme 46 Palladium-catalyzed $\gamma$ - and $\delta$-C $\left(\mathrm{sp}^{3}\right)-\mathrm{H}$ arylation of primary amines 
在瞬态导向策略中, 由亚胺和弱配位的羧酸形成的 双齿导向体系是一类高效且常用的组合，余金权课题 组 ${ }^{[58]}$ 于 2016 年报道了一种羧酸结构单元的替代物一一 2-着基吡啶，他们以 2-羟基烟醛(TDG37)为瞬态导向基, 成功实现了钯催化烷基伯胺 $\gamma-\mathrm{C}\left(\mathrm{sp}^{3}\right)-\mathrm{H}$ 键的芳基化反 应(Scheme 47). 该反应体系不但能兼容带有各种取代 基的芳基碘和杂环芳基碘，而且对于甲基、环状亚甲基 和非环状亚甲基上的 $\gamma-\mathrm{C}\left(\mathrm{sp}^{3}\right)-\mathrm{H}$ 键均适用. 同时，该反 应体系中的钯催化剂和瞬态导向基的用量还可分别降 至 $2 \%$ 和 4\%, 可谓非常高效.

Yu et al., 2016

$$
\begin{aligned}
& \text { (1) } \mathrm{Pd}(\mathrm{OPiv})_{2}(10 \mathrm{~mol} \%) \\
& \text { TDG37 }(20 \text { mol\%) } \\
& \text { AgTFA ( } 2.0 \text { equiv.) } \quad \text { Boc }-\mathrm{NH} \mathrm{Ar} \\
& \mathrm{H}_{2} \mathrm{O} \text { (10.0 equiv.) }
\end{aligned}
$$

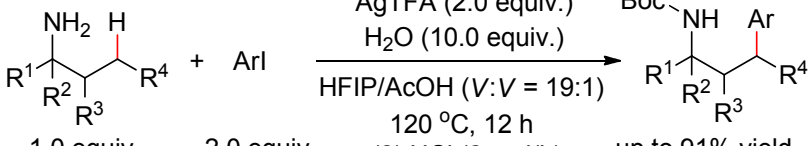

1.0 equiv. 2.0 equiv. (2) $\mathrm{HCl}(2 \mathrm{~mol} / \mathrm{L}) \quad$ up to $91 \%$ yield (3) $\mathrm{NaOH}(10 \mathrm{~mol} / \mathrm{L}), \mathrm{Boc}_{2} \mathrm{O}$

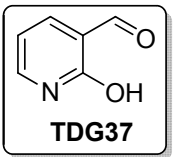

图式 47 钯催化脂肪伯胺 $\gamma-\mathrm{C}\left(\mathrm{sp}^{3}\right)-\mathrm{H}$ 键芳基化反应 Scheme 47 Palladium-catalyzed $\gamma-\mathrm{C}\left(\mathrm{sp}^{3}\right)-\mathrm{H}$ arylation of primary aliphatic amines

同样在 2017 年, Murakami 课题组 ${ }^{[59]}$ 以大位阻水杨 醛一-3,5-二叔丁基水杨醛(TDG38)为瞬态导向基，也 成功实现了烷基伯胺 $\gamma-\mathrm{C}\left(\mathrm{sp}^{3}\right)-\mathrm{H}$ 键的芳基化反应 (Scheme 48). 但是该反应的底物比较局限: (1)与氨基相

Murakami et al., 2016

(1) TDG38 (1.05 equiv.)

$\mathrm{ClCH}_{2} \mathrm{CH}_{2} \mathrm{Cl}, 110^{\circ} \mathrm{C}, 15 \mathrm{~min}$

(2) $\mathrm{Arl}\left(3.0\right.$ equiv.), $\mathrm{Pd}(\mathrm{OAc})_{2}(10 \mathrm{~mol} \%)$

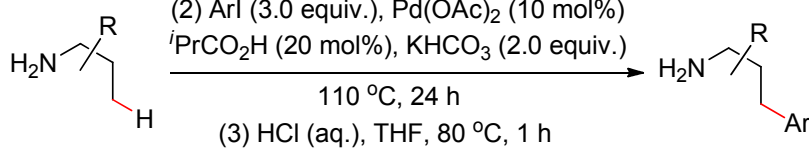

(3) $\mathrm{HCl}$ (aq.), $\mathrm{THF}, 80^{\circ} \mathrm{C}, 1 \mathrm{~h}$

1.0 equiv. (4) $\mathrm{Boc}_{2} \mathrm{O}, \mathrm{NEt}_{3}, \mathrm{CH}_{2} \mathrm{Cl}_{2}$, r.t., $1 \mathrm{~h}$ up to $78 \%$ yield

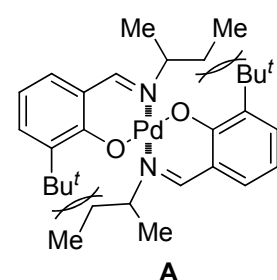
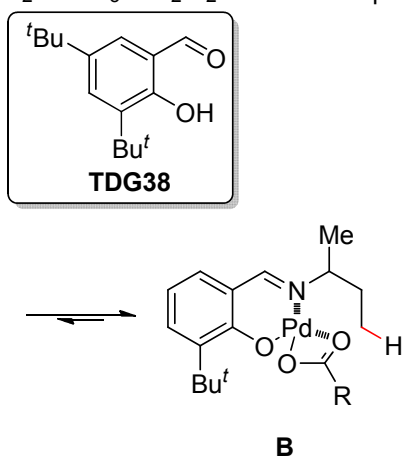

B
图式 48 钯催化脂肪伯胺 $\mathrm{C}\left(\mathrm{sp}^{3}\right)-\mathrm{H}$ 键芳基化反应 Scheme 48 Palladium-catalyzed $\mathrm{C}\left(\mathrm{sp}^{3}\right)-\mathrm{H}$ arylation of primary aliphatic amines
连的烷基必须是仲烷基；(2)需要使用略微过量的的 TDG38 进行预反应; (3)通常仅能发生甲基 C( $\left.\mathrm{sp}^{3}\right)-\mathrm{H}$ 键 的芳基化，对于更为惰性的亚甲基 $\mathrm{C}\left(\mathrm{sp}^{3}\right)-\mathrm{H}$ 键，则局 限在桥环和苄位上的亚甲基，普通链状脂肪链上的亚甲 基不反应. 他们认为大位阻水杨醛之所以是最合适的导 向基，可能是因为当使用位阻较小的水杨醛时，反应过 程中钯容易与两个亚胺底物形成中间体 $\mathbf{A}$, 从而使钯催 化剂失去反应活性; 当在水杨醛羟基的邻位引入大位阻 的叔丁基时，它与底物的碳链在空间上存在强的排斥作 用，使得该双亚胺的钯中间体不稳定，从而解离成活性 更高的单亚胺钯中间体 $\mathbf{B}$ ，进而发生碳氢键活化.

2018 年, 余金权课题组 ${ }^{[60]}$ 同样基于钯催化的瞬态导 向策略，分别以 2-着基烟醛(TDG37)、2-氯-6-差基苯甲醛 (TDG39)和 $\alpha$-酮酸类的(TDG40)为瞬态导向基，同时在 2-着弪基吡啶类配体(L1 和 L2)的促进下，成功实现了脂肪 胺的 $\gamma$-和 $\delta-\mathrm{C}\left(\mathrm{sp}^{3}\right)-\mathrm{H}$ 键的芳基化反应(Scheme 49). 值得
Yu et al., 2018

(a)

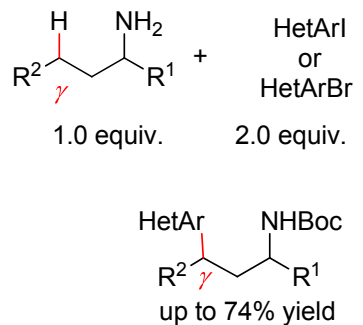

(b)

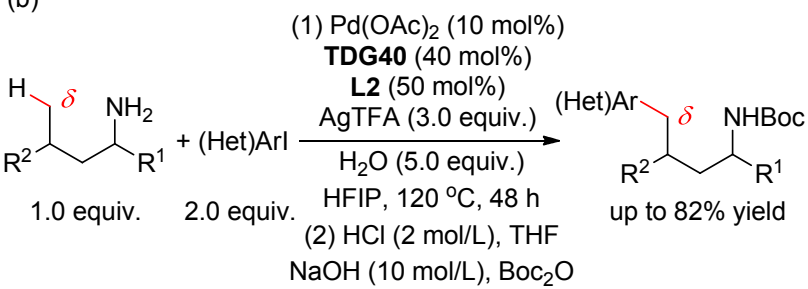

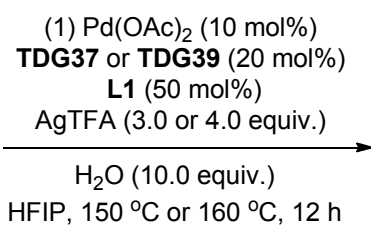

(2) $\mathrm{HCl}(2 \mathrm{~mol} / \mathrm{L}), \mathrm{THF}$ $\mathrm{NaOH}(10 \mathrm{~mol} / \mathrm{L}), \mathrm{Boc}_{2} \mathrm{O}$

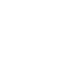


一提的是，作者认为钯与不同的瞬态导向基螯合形成的 螯合物的环系大小与经 $\gamma$-或 $\delta-\mathrm{C}\left(\mathrm{sp}^{3}\right)-\mathrm{H}$ 键活化后生成 的环钯中间体的环系大小存在一定的匹配关系, 当钯与 瞬态导向基鳌合形成六元环鳌合物时，则后续更倾向于 发生 $\gamma-\mathrm{C}\left(\mathrm{sp}^{3}\right)-\mathrm{H}$ 键活化; 而当钯与瞬态导向基鳌合形 成五元环螯合物时, 则后续更倾向于发生 $\delta-\mathrm{C}\left(\mathrm{sp}^{3}\right)-\mathrm{H}$ 键活化.

\section{3 结论}

综上所述, 基于亚胺瞬态导向策略的过渡金属催化 的碳氢键活化在近年来获得了广泛关注, 化学家们发展 了一系列高效的瞬态导向基用于醛、酮和胺类化合物的 碳氢键官能团化，已经能够实现诸如芳基化、烯基化、 炔基化、烯丙基化、氨基化、氯代、氧化等多种类型的 转化. 尤其值得一提的是, 利用手性瞬态辅基, 化学家 们也成功实现了中心手性 ${ }^{[36,49]}$ 、轴手性 ${ }^{[27,29,30,32,34]}$ 和二茂 铁面手性 ${ }^{[35]}$ 的不对称构建. 另外, 基于瞬态导向策略的 碳氢键活化也被应用于天然产物的全合成和药物分子 的后期衍生化 ${ }^{[27 b, 29 a]}$, 体现出该策略的潜在实用价值. 然而, 相较于传统的导向策略, 该领域的发展还处于初 级阶段, 仍有许多不足之处, 有待化学家们进一步探索.

相较于醛类化合物, 酮类和胺类的碳氢键活化极为 局限, 可能原因在于, 首先, 酮亚胺比醛亚胺更难形成; 其次, 存在酮亚胺与烯胺之间的互变异构; 另外, 酮亚 胺更容易受顺反异构的影响, 在反应过程中如何调节中 间体的几何结构, 让瞬态导向基将钯定位至目标碳氢 键, 极为困难. 而对于胺类化合物而言, 由于胺对金属 有很强的配位能力, 更容易形成双分子配位的金属中间 体而不利于后续反应的发生 ${ }^{[61 a]}$, 另外, 胺对许多氧化 剂和亲电试剂都比较敏感, 容易导致副反应的发 生 ${ }^{[61 \mathrm{~b}-61 \mathrm{f}]}$, 这也进一步体现在反应类型的局限性上.

目前的研究主要局限于使用贵金属, 如铑、铱和钯 等, 发展新的瞬态导向基和催化体系, 利用更廉价且低 毒的 $3 \mathrm{~d}$ 过渡金属, 如钴、镍和铜等作为催化剂, 是今后 的重要研究方向.

碳氢键活化时的位点选择性也还很单一, 对于芳香 化合物, 目前仍然局限在邻位选择性, 而对于烷基化合 物, 则主要局限于五元环金属中间体控制的脂肪醛/酮的 $\beta$ 位或脂肪胺的 $\gamma$ 位, 如何实现芳香化合物的间位和对 位的 $\mathrm{C}\left(\mathrm{sp}^{2}\right)-\mathrm{H}$ 键活化或脂肪链更远端的 $\mathrm{C}\left(\mathrm{sp}^{3}\right)-\mathrm{H}$ 键 活化 ${ }^{[62]}$, 有待化学家进一步的思考.

在立体选择性碳氢键活化方面, 目前局限于大位阻 的手性 $\alpha$-氨基酸(叔亮氨酸 $T$ TDG17 $7^{[27,29-30,32,35]}$ 和顺氨酸 TDG18 $^{[32 c]}$ )及其衍生的氨基酰胺(TDG19) ${ }^{[34,49]}$ 的手性瞬 态导向基，因此，所适用的底物局限于部分特定的醛/酮
类化合物，而对于胺类化合物瞬态导向的不对称碳氢键 活化，目前尚未报道. 对于醛/酮类化合物的不对称 $\mathrm{sp}^{3}$ 碳氢键活化，底物范围局限于邻位烷基苯甲醛类化合物 的苄位亚甲基 ${ }^{[36,49]}$ 和环丁烷酮的环上亚甲基 ${ }^{[63]}$ ，转化类 型仅限于芳基化反应. 设计和发展简单高效的新型瞬态 手性导向基，探索新的催化体系，实现底物和转化类型 更加多样性的反应, 充满着全新的挑战和机遇 ${ }^{[64]}$.

有理由相信, 随着人们对反应机理和催化模型的进 一步深入理解和认识, 这一领域必将迎来蓬勃的发展, 该策略也将为化学家们提供强有力的合成工具, 为物质 创造提供便捷的途径.

\section{References}

[1] (a) Godula, K.; Sames, D. Science 2006, 312, 67.

(b) Bergman, R. G. Nature 2007, 446, 391.

(c) Ellman, J. A.; Ackermann, L.; Shi, B. F. J. Org. Chem. 2019, 84, 12701 .

[2] For recent reviews on $\mathrm{C}-\mathrm{H}$ activation, see:

(a) Chen, X.; Engle, K. M.; Wang, D.-H.; Yu, J.-Q. Angew. Chem., Int. Ed. 2009, 48, 5094.

(b) Lyons, T. W.; Sanford, M. S. Chem. Rev. 2010, 110, 1147.

(c) Pan, F.; Shi, Z. Acta Chim. Sinica 2012, 70, 1679 (in Chinese). (潘菲, 施章杰, 化学学报, 2012, 70, 1679.)

(d) Yuan, Y.; Song, S.; Jiao, N. Acta Chim. Sinica 2015, 73, 1231 (in Chinese).

(袁逸之，宋颂，焦宁，化学学报, 2015, 73, 1231.)

(e) Liu, B.; Hu, F.; Shi, B.-F. ACS Catal. 2015, 5, 1863.

(f) Huang, Z.; Lim, H. N.; Mo, F.; Young, M. C.; Dong, G. Chem. Soc. Rev. 2015, 44, 7764.

(g) Daugulis, O.; Roane, J.; Tran, L. D. Acc. Chem. Res. 2015, 48, 1053.

(h) He, G.; Wang, B.; Nack, W. A.; Chen, G. Acc. Chem. Res. 2016 , 49,635

(i) Rao, W.-H.; Shi, B.-F. Org. Chem. Front. 2016, 3, 1028.

(j) Yang, Y.; Lan, J.; You, J. Chem. Rev. 2017, 117, 8787.

(k) He, J.; Wasa, M.; Chan, K. S. L.; Shao, Q.; Yu, J.-Q. Chem. Rev. 2017, 117, 8754 .

(1) Huang, J.; Gu, Q.; You, S.-L. Chin. J. Org. Chem. 2018, 38, 51 (in Chinese)

(黄家翩，顾庆，游书力，有机化学, 2018, 38, 51.)

(m) Ren, Q.; Nie, B.; Zhang, Y.; Zhang, J. Chin. J. Org. Chem. 2018, 38, 2465 (in Chinese)

(任青云, 聂蓫, 张英俊, 张雯, 有机化学, 2018, 38, 2465.)

(n) Zhao, K.; Yang, L.; Liu, J.; Xia, C. R. Chin. J. Org. Chem. 2018, 38, 2833 (in Chinese).

(赵康，杨䂞，刘建华，夏春谷，有机化学, 2018, 38, 2833.)

(o) Xu, L.; Xu, H.; Lin, H.; Dai, H. Chin. J. Org. Chem. 2018, 38, 1940 (in Chinese).

(徐琳琳, 徐辉, 林海霞, 戴辉雄, 有机化学, 2018, 38, 1940.)

(p) Zhan, B.-B.; Shi, B.-F. Chin. J. Org. Chem. 2019, 39, 3602 (in Chinese).

(占贝贝，史炳锋，有机化学, 2019, 39, 3602.)

(q) Gandeepan, P.; Müller, T.; Zell, D.; Cera, G.; Warratz, S.; Ackermann, L. Chem. Rev. 2019, 119, 2192.

(r) Han, Y.-Q.; Zhou, T. Chin. J. Chem. 2020, 38, 527.

(s) Liu, Y.-H.; Xia, Y.-N.; Shi, B.-F. Chin. J. Chem. 2020, 38, 635.

[3] (a) Zhang, M.; Zhang, Y.; Jie, X.; Zhao, H.; Li, G.; Su, W. Org. Chem. Front. 2014, 1, 843.

(b) Sambiagio, C.; Schönbauer, D.; Blieck, R.; Dao-Huy, T.; Pototschnig, G.; Schaaf, P.; Wiesinger, T.; Zia, M. F.; Wencel-Delord, J.; Besset, T.; Maes, B. U. W.; Schnürch, M. Chem. Soc. Rev. 2018, 47, 6603 . 
(c) Zhang, Q.; Shi, B.-F. Chin. J. Chem. 2019, 37, 647.

(d) Rej, S.; Ano, Y.; Chatani, N. Chem. Rev. 2020, 120, 1788.

[4] (a) Besset, T.; Zhao, Q.; Poisson, T.; Pannecoucke, X. Synthesis 2017, 49, 4808 .

(b) Qin, Y.; Zhu, L.; Luo, S. Chem. Rev. 2017, 117, 9433.

(c) Kim, D.-S.; Park, W.-J.; Jun, C.-H. Chem. Rev. 2017, 117, 8977

(d) Gandeepan, P.; Ackermann, L. Chem 2018, 4, 199.

(e) Bhattacharya, T.; Pimparkar, S.; Maiti, D. RSC Adv. 2018, 8, 19456.

(f) St John-Campbell, S.; Bull, J. A. Org. Biomol. Chem. 2018, 16, 4582 .

(g) Liao, G.; Wu, Y.-J.; Shi, B.-F. Acta Chim. Sinica 2020, 78, 289 (in Chinese).

(廖港，吴勇杰，史炳锋，化学学报, 2020, 78, 289.)

[5] For selected examples of phosphites as transient directing groups, see: (a) Bedford, R. B.; Coles, S. J.; Hursthouse, M. B.; Limmert, M. E. Angew. Chem., Int. Ed. 2003, 42, 112.

(b) Bedford, R. B.; Limmert, M. E. J. Org. Chem. 2003, 68, 8669

(c) Bedford, R. B.; Betham, M.; Caffyn, A. J.; Charmant, J. P.; Lewis-Alleyne, L. C.; Long, P. D.; Polo-Ceron, D.; Prashar, S. Chem. Commun. 2008, 990.

(d) Bedford, R. B.; Haddow, M. F.; Webster, R. L.; Mitchell, C. J. Org. Biomol. Chem. 2009, 7, 3119.

(e) Carrion, M. C.; Cole-Hamilton, D. J. Chem. Commun. 2006, 4527 .

(f) Lewis, J. C.; Wu, J.; Bergman, R. G.; Ellman, J. A. Organometallics 2005, 24, 5737.

(g) Oi, S.; Watanabe, S.-I.; Fukita, S.; Inoue, Y. Tetrahedron Lett. 2003, 44, 8665 .

(h) Yang, J. F.; Wang, R. H.; Wang, Y. X.; Yao, W. W.; Liu, Q. S.; Ye, M. Angew. Chem., Int. Ed. 2016, 55, 14116.

(i) Liu, Q.-S.; Wang, D.-Y.; Yang, J.-F.; Ma, Z.-Y.; Ye, M. Tetrahedron 2017, 73, 3591 .

[6] Mo, F.; Dong, G. Science 2014, 345, 68.

[7] For selected examples of transient enamine directing groups, see: (a) Wang, Z.; Reinus, B. J.; Dong, G. J. Am. Chem. Soc. 2012, 134, 13954.

(b) Mo, F.; Lim, H. N.; Dong, G. J. Am. Chem. Soc. 2015, 137, 15518.

(c) Xu, Y.; Young, M. C.; Dong, G. J. Am. Chem. Soc. 2017, 139, 5716 .

(d) Lim, H. N.; Dong, G. Angew. Chem., Int. Ed. 2015, 54, 15294

(e) Xu, Y.; Su, T.; Huang, Z.; Dong, G. Angew. Chem., Int. Ed. 2016, 55,2559 .

[8] Jun, C.-H.; Lee, H.; Hong, J.-B. J. Org. Chem. 1997, 62, 1200.

[9] Jun, C.-H.; Lee, D.-Y.; Hong, J.-B. Tetrahedron Lett. 1997, 38, 6673.

[10] Vautravers, N. R.; Regent, D. D.; Breit, B. Chem. Commun. 2011, 47,6635 .

[11] Beletskiy, E. V.; Sudheer, C.; Douglas, C. J. J. Org. Chem. 2012, 77,5884 .

[12] Jun, C.-H.; Moon, C. W.; Hong, J.-B.; Lim, S.-G.; Chung, K.-Y.; Kim, Y.-H. Chem.-Eur. J. 2002, 8, 485.

[13] Kuninobu, Y.; Nishina, Y.; Shouho, M.; Takai, K. Angew. Chem., Int. Ed. 2006, 45, 2766.

[14] Chen, S.; Yu, J.; Jiang, Y.; Chen, F.; Cheng, J. Org. Lett. 2013, 15, 4754

[15] Tan, P. W.; Juwaini, N. A. B.; Seayad, J. Org. Lett. 2013, 15, 5166.

[16] Li, G.; Jiang, J.; Xie, H.; Wang, J. Chem.-Eur. J. 2019, 25, 4688.

[17] Liu, X. H.; Park, H.; Hu, J. H.; Hu, Y.; Zhang, Q. L.; Wang, B. L.; Sun, B.; Yeung, K. S.; Zhang, F. L.; Yu, J. Q. J. Am. Chem. Soc. 2017, 139, 888

[18] Chen, X. Y.; Ozturk, S.; Sorensen, E. J. Org. Lett. 2017, 19, 1140.

[19] Xu, J.; Liu, Y.; Wang, Y.; Li, Y.; Xu, X.; Jin, Z. Org. Lett. 2017, 19, 1562 .

[20] Hu, W.; Zheng, Q.; Sun, S.; Cheng, J. Chem. Commun. 2017, 53, 6263.

[21] Li, B.; Seth, K.; Niu, B.; Pan, L.; Yang, H.; Ge, H. Angew. Chem.,
Int. Ed. 2018, 57, 3401.

[22] Wang, D. Y.; Guo, S. H.; Pan, G. F.; Zhu, X. Q.; Gao, Y. R.; Wang, Y. Q. Org. Lett. 2018, 20, 1794

[23] Wang, Y. F.; Xu, W. G.; Sun, B.; Yu, Q. Q.; Li, T. J.; Zhang, F. L. J. Org. Chem. 2019, 84, 13104.

[24] Chen, X. Y.; Sorensen, E. J. J. Am. Chem. Soc. 2018, 140, 2789.

[25] (a) Li, F.; Zhou, Y.; Yang, H.; Liu, D.; Sun, B.; Zhang, F. L. Org. Lett. 2018, 20, 146.

(b) Zhan, B.-B.; Li, Y.; Xu, J.-W.; Nie, X.-L.; Fan, J.; Jin, L.; Shi, B.-F. Angew. Chem., Int. Ed. 2018, 57, 5858.

[26] (a) Baudoin, O. Eur. J. Org. Chem. 2005, 4223.

(b) Bringmann, G.; Price Mortimer, A. J.; Keller, P. A.; Gresser, M. J.; Garner, J.; Breuning, M. Angew. Chem., Int. Ed. 2005, 44, 5384.

(c) Wencel-Delord, J.; Panossian, A; Leroux, F. R.; Colobert, F. Chem. Soc. Rev. 2015, 44, 3418.

(d) Ma, G.; Sibi, M. P. Chem.-Eur. J. 2015, 21, 11644.

(e) Kumarasamy, E.; Raghunathan, R.; Sibi, M. P.; Sivaguru, J Chem. Rev. 2015, 115, 11239.

(f) Mori, K.; Itakura, T.; Akiyama, T. Angew. Chem., Int. Ed. 2016, $55,11642$.

(g) Xu, C.; Zheng, H.; Hu, B.; Liu, X.; Liu, L.; Feng, X. Chem. Commun. 2017, 53, 9741.

(h) Zilate, B.; Castrogiovanni, A.; Sparr, C. ACS Catal. 2018, 8, 2981.

(i) Liao, G.; Zhou, T.; Yao, Q.-J.; Shi, B.-F. Chem. Commun. 2019, 55,8514 .

[27] (a) Yao, Q. J.; Zhang, S.; Zhan, B. B.; Shi, B. F. Angew. Chem., Int. Ed. 2017, 56, 6617.

(b) Fan, J.; Yao, Q. J.; Liu, Y. H.; Liao, G.; Zhang, S.; Shi, B. F. Org. Lett. 2019, 21, 3352.

[28] (a) Wang, Q.; Gu, Q.; You, S.-L. Angew. Chem., Int. Ed. 2019, 58, 6818.

(b) Chen, J.; Liu, Y. E.; Gong, X.; Shi, L.; Zhao, B. Chin. J. Chem. 2019, 37, 103.

(c) Gong, L.-Z. Sci. China: Chem. 2019, 62, 3.

(d) Li, S.; Chen, X.-Y.; Enders, D. Chem 2018, 4, 2026

[29] (a) Liao, G.; Yao, Q. J.; Zhang, Z. Z.; Wu, Y. J.; Huang, D. Y.; Shi, B. F. Angew. Chem., Int. Ed. 2018, 57, 3661.

(b) Liao, G.; Li, B.; Chen, H. M.; Yao, Q. J.; Xia, Y. N.; Luo, J.; Shi, B. F. Angew. Chem., Int. Ed. 2018, 57, 17151.

[30] Liao, G.; Chen, H. M.; Xia, Y. N.; Li, B.; Yao, Q. J.; Shi, B. F. Angew. Chem., Int. Ed. 2019, 58, 11464.

[31] Zhang, S.; Liao, G.; Shi, B. Chin. J. Org. Chem. 2019, 39, 1522 (in Chinese).

(张硕, 廖港, 史炳锋, 有机化学, 2019, 39, 1522.)

[32] (a) Zhang, S.; Yao, Q.-J.; Liao, G.; Li, X.; Li, H.; Chen, H.-M.; Hong, X.; Shi, B.-F. ACS Catal. 2019, 9, 1956.

(b) Chen, H.-M.; Zhang, S.; Liao, G.; Yao, Q.-J.; Xu, X.-T.; Zhang, K.; Shi, B.-F. Organometallics 2019, 38, 4022.

(c) Zhang, J.; Xu, Q.; Wu, J.; Fan, J.; Xie, M. Org. Lett. 2019, 21, 6361.

[33] Jin, L.; Yao, Q.-J.; Xie, P.-P.; Li, Y.; Zhan, B.-B.; Han, Y.-Q.; Hong, X.; Shi, B.-F. Chem 2020, 6, 497

[34] Song, H.; Li, Y.; Yao, Q. J.; Jin, L.; Liu, L.; Liu, Y. H.; Shi, B. F. Angew. Chem., Int. Ed. 2020, 59, 6576.

[35] Xu, J.; Liu, Y.; Zhang, J.; Xu, X.; Jin, Z. Chem. Commun. 2018, 54, 689.

[36] Zhang, F.-L.; Hong, K.; Li, T.-J.; Park, H.; Yu, J.-Q. Science 2016, $351,252$.

[37] (a) Ma, F.; Lei, M.; Hu, L. Org. Lett. 2016, 18, 2708. (b) Chen, J.; Bai, C.; Ma, H.; Liu, D.; Bao, Y.-S. Chin. Chem. Lett 2020, DOI: $10.1016 /$ j.cclet.2020.02.055.

[38] Park, H.; Yoo, K.; Jung, B.; Kim, M. Tetrahedron 2018, 74, 2048.

[39] Tang, M.; Yu, Q.; Wang, Z.; Zhang, C.; Sun, B.; Yi, Y.; Zhang, F. L. Org. Lett. 2018, 20, 7620.

[40] Yang, K.; Li, Q.; Liu, Y.; Li, G.; Ge, H. J. Am. Chem. Soc. 2016, $138,12775$.

[41] St John-Campbell, S.; White, A. J. P.; Bull, J. A. Chem. Sci. 2017 
8,4840 .

[42] Hong, K.; Park, H.; Yu, J. Q. ACS Catal. 2017, 7, 6938.

[43] Pan, L.; Yang, K.; Li, G.; Ge, H. Chem. Commun. 2018, 54, 2759.

[44] Zhang, Y.-F.; Wu, B.; Shi, Z.-J. Chem.-Eur. J. 2016, 22, 17808.

[45] Mu, D.; Wang, X.; Chen, G.; He, G. J. Org. Chem. 2017, 82, 4497.

[46] Wang, X.; Song, S.; Jiao, N. Chin. J. Chem. 2018, 36, 213.

[47] Rasheed, O. Synlett 2018, 29, 1033.

[48] Huang, J.; Ding, J.; Ding, T. M.; Zhang, S.; Wang, Y.; Sha, F.; Zhang, S. Y.; Wu, X. Y.; Li, Q. Org. Lett. 2019, 21, 7342.

[49] Park, H.; Verma, P.; Hong, K.; Yu, J. Q. Nat. Chem. 2018, 10, 755.

[50] Chen, X. Y.; Ozturk, S.; Sorensen, E. J. Org. Lett. 2017, 19, 6280.

[51] Li, F.; Zhou, Y.; Yang, H.; Wang, Z.; Yu, Q.; Zhang, F.-L. Org. Lett. 2019, 21, 3692.

[52] Yong, Q.; Sun, B.; Zhang, F.-L. Tetrahedron Lett. 2019, 60, 151263.

[53] Qiao, H.; Sun, B.; Yu, Q.; Huang, Y.-Y.; Zhou, Y.; Zhang, F.-L. Org. Lett. 2019, 21, 6914.

[54] Wu, Y.-J.; Yao, Q.-J.; Chen, H.-M.; Liao, G.; Shi, B.-F. Sci. China: Chem. 2020, 63, 875 .

[55] Liu, Y.; Ge, H. Nat. Chem. 2016, 9, 26.

[56] (a) Xu, J.-W.; Zhang, Z.-Z.; Rao, W.-H.; Shi, B.-F. J. Am. Chem. Soc. 2016, 138, 10750.

(b) Zhan, B.-B.; Li, Y.; Xu, J.-W.; Nie, X.-L.; Fan, J.; Jin, L.; Shi, B.-F. Angew. Chem., Int. Ed. 2018, 57, 5858.

[57] Xu, Y.; Young, M. C.; Wang, C.; Magness, D. M.; Dong, G. Angew. Chem., Int. Ed. 2016, 55, 9084.

[58] Wu, Y.; Chen, Y. Q.; Liu, T.; Eastgate, M. D.; Yu, J. Q. J. Am. Chem. Soc. 2016, 138, 14554.

[59] Yada, A.; Liao, W.; Sato, Y.; Murakami, M. Angew. Chem., Int. Ed.
2017, 56, 1073.

[60] Chen, Y.-Q.; Wang, Z.; Wu, Y.; Wisniewski, S. R.; Qiao, J. X.; Ewing, W. R.; Eastgate, M. D.; Yu, J.-Q. J. Am. Chem. Soc. 2018 , 140, 17884.

[61] (a) Vicente, J.; Saura-Llamas, I.; Palin, M. G.; Jones, P. G.; Ramírez de Arellano, M. C. Organometallics 1997, 16, 826.

(b) Largeron, M. Eur. J. Org. Chem. 2013, 2013, 5225.

(c) Corey, E. J.; Achiwa, K. J. Am. Chem. Soc. 1969, 91, 1429.

(d) Hartwig, J. F.; Richards, S.; Barañano, D.; Paul, F. J. Am. Chem. Soc. 1996, 118, 3626.

(e) Wolfe, J. P.; Wagaw, S.; Buchwald, S. L. J. Am. Chem. Soc. 1996, 118,7215 .

(f) Ryland, B. L.; Stahl, S. S. Angew. Chem., Int. Ed. 2014, 53, 8824.

[62] (a) Liu, L.; Liu, Y.-H.; Shi, B.-F. Chem. Sci. 2020, 11, 290.

(b) Xu, J.-W.; Zhang, Z.-Z.; Rao, W.-H.; Shi, B.-F. J. Am. Chem. Soc. 2016, 138, 10750.

[63] Xiao, L.-J.; Hong, K.; Luo, F.; Hu, L.; Ewing, W. R.; Yeung, K.-S.; Yu, J.-Q. Angew. Chem., Int. Ed. 2020, 59, 9594.

[64] (a) Chen, G.; Gong, W.; Zhuang, Z.; Andrä, M. S.; Chen, Y.-Q.; Hong, X.; Yang, Y.-F.; Liu, T.; Houk, K. N.; Yu, J.-Q. Science 2016, 353, 1023.

(b) Yan, S.-Y.; Han, Y.-Q.; Yao, Q.-J.; Nie, X.-L.; Liu, L.; Shi, B.-F. Angew. Chem., Int. Ed. 2018, 57, 9093.

(c) Han, Y.-Q.; Ding, Y.; Zhou, T.; Yan, S.-Y.; Song, H.; Shi, B.-F. J. Am. Chem. Soc. 2019, 141, 4558

(d) Zhou, T.; Jiang, M.-X.; Yang, X.; Yue, Q.; Han, Y.-Q.; Ding, Y.; Shi, B.-F. Chin. J. Chem. 2020, 38, 242.

(Cheng, F.) 